\author{
Giorgio BALDIZZONE* - Stefano SCALERCIO**
}

\title{
Contributo alla conoscenza dei microlepidotteri dell'Aspromonte (Lepidoptera)
}

\begin{abstract}
Riassunto: In questo lavoro vengono riportati i risultati di una settimana di raccolta di microlepidotteri effettuata con diverse tecniche in Calabria, più precisamente sull'Aspromonte e alla foce della Fiumara Amendolea. Sono state rinvenute 159 specie di cui Eana nervana (Tortricidae) e Merrifeldia garrigae (Pterophoridae) nuove per la fauna italiana, Opogona omoscopa (Tineidae), Triberta cistifoliella (Gracillariidae), Ornativalva heluanensis e Neophriseria singula (Gelechiidae) segnalate per la prima volta per l'Italia continentale, oltre a 8 specie nuove per l'Italia centromeridionale, 16 per l'Italia meridionale, e 28 segnalate per la prima volta in Calabria. La grande quantità di novità faunistiche sono l'evidenza delle scarse conoscenze disponibili sulla fauna di questi insetti per l'Italia in generale e per le regioni meridionali in particolare.
\end{abstract}

Abstract: Contribution to the knowledge of the microlepidoptera of the Aspromonte Massif (Lepidoptera)

In this paper we reported the results of a week of microlepidoptera samplings carried out using several collecting methods in Calabria region, precisely on the Aspromonte Massif and at the Fiumara Amendolea mouth, southern Italy. We found 159 species among which Eana nervana (Tortricidae) and Merrifeldia garrigae (Pterophoridae) are new for the Italian fauna, Opogona omoscopa (Tineidae), Triberta cistifoliella (Gracillariidae), Ornativalva heluanensis and Neophriseria singula (Gelechiidae) are new for the fauna of continental Italy. Furthermore, 8 species are new for peninsular Italy, 16 for southern Italy, and 28 for the Calabria region. So many faunistic novelties are due to the scarce knowledge on microlepidoptera for Italy in general and for southern Italy in particular.

Key words: Eana nervana, Merrifeldia garrigae, fauna, Parco Nazionale dell’Aspromonte, Calabria.

\section{INTRODUZIONE}

La fauna dei microlepidotteri dell'Italia meridionale continentale è stata molto poco esplorata e $\mathrm{i}$ primi dati, e per molto tempo gli unici, sono da riferire a Costa O.G. (1832-1836) per il Regno di Napoli il quale molto spesso però riporta solo genericamente la presenza nel Regno. A questo lavoro sono seguiti quelli di Costa A. $(1863,1881)$ che ha indagato la fauna entomologica della Calabria Ulteriore, in parte corrispondente all'Aspromonte e ai suoi dintorni, citando anche alcune specie di microlepidotteri. Le conoscenze sulle regioni meridionali sono state incrementate molto poco fino ai lavori di Zangheri (1955a,b, 1960) che aveva esplorato alcune limitate aree del meridione. Successivamente, si ricordano i lavori di Ippolito (1987) sui Pyraloidea dell'Italia meridionale, nel quale sono citate un numero limitato di specie, e di Scalercio (2009) sugli Psychidae della sola Calabria. Ad oggi gli unici lavori organici sulla fauna italiana in cui è possibile avere informazioni relative alla distribuzione dei microlepidotteri anche in Italia meridionale sono dedicati ai Tortricidae (Trematerra,
2003, 2004), ai Pterophoridae (Prola \& Racheli, 1984) e ai Coleophoridae (Baldizzone, 2018), mentre per le restanti famiglie le conoscenze sulla distribuzione al meridione sono limitate alle macro-aree individuate nella checklist delle specie della fauna italiana (Minelli et al., 1995) e ai ben più datati cataloghi di Curò (1880, 1883) e Mariani (1943).

I pochi dati disponibili per l'Aspromonte sono quasi tutti riconducibili ad Hartig, il quale ha raccolto molto materiale negli anni a cavallo fra fine anni '60 e inizi anni ' 70 dello scorso secolo intorno a Gambarie, pubblicandone solo una minima parte (Hartig, 1972). Tuttavia, le date di raccolta di alcune segnalazioni relative al territorio dell'Aspromonte non lasciano dubbi sulla paternità del dato. Sono questi i casi di Rhigognostis incarnatella (Steudel, 1873) (Plutellidae), Aroga velocella (Zeller, 1839) (Gelechiidae), e Pterolonche inspersa Staudinger, 1859 (Pterolonchidae) in Parenti (2000), e di Pseudatemelia pallidella Jäckh 1972 (Lypusidae) in Jäckh (1972). Anche la recente descrizione di una nuova specie di Coleophoridae, probabilmente endemica dell'Aspromonte,

\footnotetext{
*Giorgio Baldizzone, Scuola di Biodiversità WWF “Villa Paolina”, loc. Valmanera 94, 14100 Asti (AS), Italia. E-mail: baldizzonegiorgio@gmail.com ${ }^{* *}$ Stefano Scalercio, Consiglio per la ricerca in Agricoltura e l'Analisi dell'Economia Agraria, Centro di Ricerca Foreste e Legno, Contrada Li Rocchi, Rende (CS), Italia. E-mail: stefano.scalercio@crea.gov.it
} 
Coleophora aspromontis Baldizzone, 2014, ha avuto origine da esemplari della collezione Hartig, che uno degli autori (GB) ha individuato come appartenenti ad una specie non ancora descritta parecchi decenni dopo la loro raccolta.

Con questo lavoro si intende dare un contributo alla conoscenza della fauna di un territorio molto poco esplorato, ma di estremo interesse biogeografico come è il comprensorio dell'Aspromonte.

\section{Materiali e Metodi}

La ricerca svolta in Aspromonte aveva come scopo prioritario il reperimento di esemplari della nuova specie di Coleophoridae, di cui Giorgio Baldizzone conosceva pochi esemplari raccolti da Hartig nel 1971 nei dintorni di Gambarie.

Le attività di raccolta sono state effettuate dal 6 al 12 luglio 2013 in sette diverse località della provincia di Reggio Calabria, soprattutto nei dintorni di Gambarie, nel comune di Santo Stefano in Aspromonte (RC) (Fig. 1). Pochi dati si riferiscono a rac- colte dirette effettuate durante il giorno, mentre la maggior parte degli esemplari è stata raccolta di notte utilizzando come fonte luminosa una lampada da $160 \mathrm{~W}$ a luce miscelata alimentata con un generatore portatile di corrente elettrica, posizionata davanti ad un telo bianco sul quale sono stati prelevati gli insetti. Oltre a questa, in alcune notti è stata utilizzata una struttura a piramide nella quale erano collocati due tubi da $15 \mathrm{~W}$ a luce attinica. Per tre notti sono state messe in funzione anche 5 trappole, che montavano un tubo attinico da $18 \mathrm{~W}$ oppure due tubi da $18 \mathrm{~W}$ di cui uno attinico e uno di Wood.

Tutti gli esemplari sono stati raccolti e preparati da Giorgio Baldizzone il giorno successivo alla cattura, secondo la tecnica tradizionale per i microlepidotteri.

Di seguito si riporta l'elenco completo delle località indagate riportando per ognuna di esse il toponimo, il comune in cui ricade, la quota sul livello del mare, le coordinate geografiche in forma decimale e una brevissima descrizione dell'ambiente:

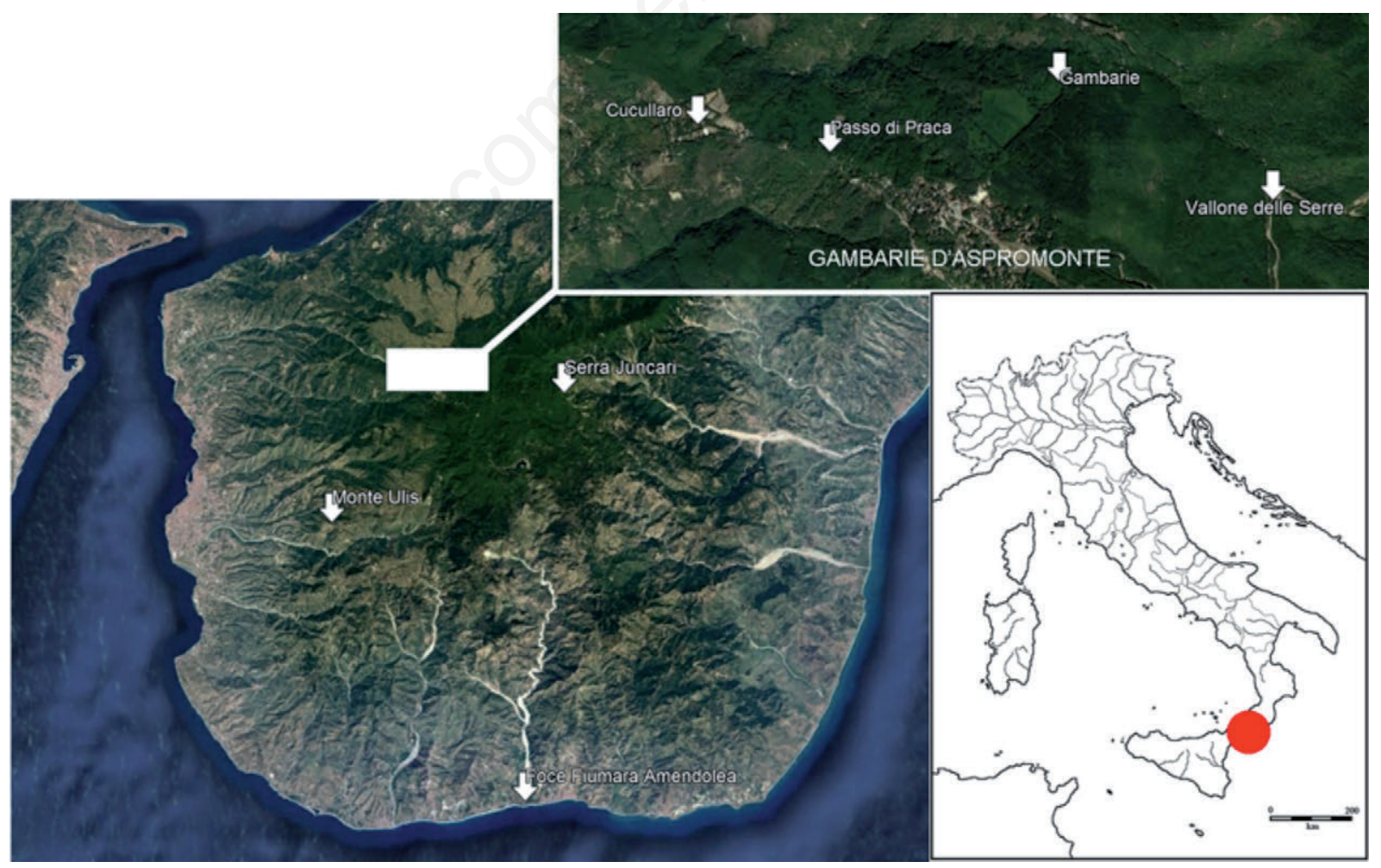

Fig. 1. Localizzazione delle località di raccolta. 
1. Passo di Praca, Santo Stefano in Aspromonte (RC); 1210m, 38,1707N, 15,8269E; castagneto;

2. Monte Ulis, Cardeto (RC); 1045m; 38,0941N, $15,7558 \mathrm{E}$; querceto;

3. Foce Fiumara Amendolea, Condofuri Marina (RC); 5m; 37,9288N, 15,8888E; gariga golenale;

4. Gambarie, Santo Stefano in Aspromonte (RC); $1340 \mathrm{~m} ; 38,1744 \mathrm{~N}, 15,8447 \mathrm{E}$; pineta;

5. Cucullaro, Santo Stefano in Aspromonte (RC); $1160 \mathrm{~m} ; 38,1727 \mathrm{~N}, 15,8166 \mathrm{E}$; margine di bosco misto di latifoglie;

6. Vallone delle Serre, Santo Stefano in Aspromonte (RC); 1430m; 38,1669N, 15,8602E; radura in faggeta;

7. Serra Juncari, Roghudi (RC); $1750 \mathrm{~m} ; 38,1625 \mathrm{~N}$, $15,9277 \mathrm{E}$; radura in faggeta.

L'elenco delle specie segue in linea di massima l'ordinamento di Karsholt \& Razowsky (1996) con alcune modifiche alla luce di recenti pubblicazioni, mentre la nomenclatura è conforme a quella più aggiornata riportata in Fauna Europaea (Karsholt \& Nieukerken, 2017), database utilizzato anche per verificare le distribuzioni geografiche (ultimo accesso 09 maggio 2018).

Per ogni specie si riportano località e data di rinvenimento. Il metodo di cattura è indicato con (lux) quando la specie è stata attratta con la lampada o con trappole, in tutti gli altri casi ci si riferisce a cattura diretta. Tranne quando diversamente indicato, il materiale è conservato nella collezione Baldizzone. Per tutte le specie sono riportate le informazioni disponibili sulla distribuzione geografica generale e quella italiana. Vengono anche fornite brevi note biologiche per ogni specie.

\section{Elenco delle specie}

\section{Micropterigidae}

Micropterix myrtetella Zeller, 1850 [det. Karsholt] Passo di Praca, 6.VII.2013 (lux); Monte Ulis, 7-8, 1011.VII.2013 (lux).

DistribuZione Geografica: Italia, Austria, Ungheria, Croazia, Macedonia, Romania, Bulgaria, Albania, Grecia; probabilmente questi dati sono conseguenti a confusioni con altre specie (Zeller-Lukashort et al., 2007). In Italia era segnalata per il locus typicus (Toscana) e genericamente per il Sud
(Whitebread, 1995), ma anche in questo caso sono possibili errori identificativi. Prima segnalazione per la Calabria.

Biologia: Sconosciuta.

\section{Nepticulidae [det. van Nieukerken]}

Stigmella samiatella (Zeller, 1839)

Monte Ulis, 7-8, 10-11.VII.2013 (lux).

DistribuZIONE GEOGRAFICA: Largamente diffusa in Europa, Turchia e Georgia. In Italia è segnalata di Piemonte, Trentino-Alto Adige, Liguria, Basilicata, $\mathrm{Pu}-$ glia, Calabria e Sicilia (Baldizzone et al., 2013).

Biologia: Larva su Castanea sativa e Quercus di varie specie.

Trifurcula calycotomella A. \& Z. Laštuvka, 1997 Monte Ulis, 10-11.VII.2013 (lux).

DisTRIBUZIONE GEOGRAFICA: Specie descritta in base a esemplari raccolti in Liguria, è conosciuta anche di Spagna, Francia, Croazia, Grecia, Turchia, Tunisia. Prima segnalazione per la Calabria.

Biologia: Larva su Calycotome spinosa.

Ectoedemia liebwerdella Zimmermann, 1940

Monte Ulis, 7, 11.VII.2013 (lux).

DistribuZIONE GEOGRAFICA: Diffusa in Europa continentale, Turchia e Russia, è nota in Italia per Piemonte, Umbria, Lazio e Abruzzo (Baldizzone et al., 2013). Prima segnalazione per l'Italia meridionale.

Biologia: Larva nella corteccia di Fagus sylvatica e probabilmente anche di alcune specie di Quercus.

Ectoedemia caradjai (Groschke, 1944)

Monte Ulis, 10.VII.2013 (lux).

DistRIBUZIONE GEOGRAFICA: Largamente diffusa in Europa meridionale, dal Portogallo alla Turchia e Russia, nel Nord fino alla Repubblica Ceca. In Italia segnalata di tutta la penisola e della Sicilia (regione di cui è stata descritta) (Baldizzone et al., 2013).

Biologia: Larva su varie specie di Quercus, particolarmente $Q$. pubescens.

Ectoedemia heringella (Mariani, 1939)

Monte Ulis, 8.VII.2013 (lux).

DistribuZIONE GeOgRAFICA: Europa meridionale: Francia di Sud-Est, Corsica, Italia, Croazia, Grecia, Cipro e probabilmente Tunisia; recentemente introdotta in Inghilterra. In Italia segnalata di tutta la peni- 
sola, della Sicilia (regione di cui è stata descritta) e della Sardegna (Baldizzone et al., 2013).

Biologia: Larva su Quercus ilex, Q. alnifolia e probabilmente su specie di Quercus con foglie decidue, come $Q$. pubescens e $Q$. petraea

\section{Tischeriidae}

Tischeria decidua Wocke, 1876 [det. Triberti]

Monte Ulis, 7.VII.2013 (lux).

DistribuZIONE GEOGRAFICA: Europa centro-meridionale. In Italia la specie è piuttosto comune, non ancora segnalata per la Sardegna (Baldizzone et al., 2013). Biologia: La larva mina le foglie di Castanea sativa, Quercus spp. (solo caducifolie).

\section{Tineidae}

Crassicornella crassicornella (Zeller, 1847)

Monte Ulis, 8 e 10.VII.2013 (lux).

DISTRIBUZIONE GEOGRAFICA: Italia meridionale, Sicilia, Albania, Grecia, Creta, Turchia. Prima segnalazione per la Calabria.

BIOLOGIA: Non conosciuta. Forse si sviluppa nei detriti delle tane di piccoli mammiferi.

Cephimallota angusticolella (Zeller, 1839) [det. Nel] Monte Ulis, 8 e 10.VII.2013 (lux).

DisTRIBUZIONE GEOGRAFICA: La specie ha una distribuzione vicariante con quella di Cephimallota crassiflavella (Zeller, 1852). Le due specie s'incontrano lungo una linea che attraversa l'Italia centrale, la Croazia, l'Ungheria, la Slovacchia, e crassiflavella estende il suo areale a Ovest di questa linea, mentre angusticostella si distribuisce a Est e Sud della medesima. In Italia le due specie volano insieme in Piemonte (Parco Capanne di Marcarolo) (Baldizzone et al., 2013) e sul Monte Pollino in Basilicata (Gaedike \& Mally, 2011). Segnalata in Calabria per Fiumicella di Tortora (Gaedike \& Mally, 2011).

Biologia: Sconosciuta. Forse la larva vive negli scavi del terreno prodotti da imenotteri o nelle loro rosure.

Infurcitinea parentii G. Petersen, 1964 [det. Nel] Passo di Praca, 6.VII.2013 (lux); Monte Ulis, 11.VII.2013 (lux).

DistribuZIONE GEOGRAFICA: Fino a oggi la specie è conosciuta solo di poche località di Spagna, Francia e Italia. (Baldizzone et al., 2013). Prima segnalazione per l'Italia meridionale.
Biologia: Sconosciuta. La larva potrebbe essere lichenofaga.

Infurcitinea finalis Gozmány, 1959

Vallone delle Serre, 7.VII.2013 (lux); Monte Ulis, 10.VII.2013 (lux).

DistribuZione GEOGRAFICA: Spagna, Francia meridionale, Italia, Austria, Slovacchia, Ungheria, Russia meridionale, Romania, Bulgaria, Grecia, Turchia. In Italia è conosciuta di Piemonte, Trentino, Friuli, Toscana, Molise, Basilicata e Calabria (Baldizzone et al., 2013).

Biologia: Sconosciuta. La larva potrebbe essere lichenofaga. L'adulto in Ungheria è stato attratto con feromoni.

Morophaga choragella (Denis \& Schiffermüller, 1775)

Vallone delle Serre, 7.VII.2013 (lux).

DisTRIBUZIONE GEOGRAFICA: Dall'Estremo Oriente attraverso tutta l'Europa fino alla Spagna e al Portogallo, Grecia settentrionale, Turchia occidentale. In Italia è conosciuta di numerose località del Nord, con diverse segnalazioni per l'Appennino fino alla Calabria e all'Aspromonte; è nota anche della Sardegna (Baldizzone et al., 2013).

Biologia: Le larve vivono nel legno marcio e nei funghi arboricoli crescenti su betulla, pioppo, tiglio, quercia, faggio, ontano, abete bianco.

Nemapogon variatella (Clemens, 1859) [det. Nel] Passo Praca, 6.VII.2013 (lux).

DistribuZione geografica: Conosciuta di tutta l'Europa, dell'Africa del Nord e dall'Asia Minore fino all'Estremo Oriente. Presente nella Regione Neartica, è stata diffusa in molte regioni come parassita dei cereali. Ampiamente presente in tutta Italia (Baldizzone et al., 2013).

Biologia: La larva vive normalmente in diversi funghi arboricoli.

Niditinea striolella (Matsumura, 1931) [det. Nel] Passo Praca, 6.VII.2013 (lux).

Distribuzione GeOgRafica: Conosciuta di tutta l'Europa, dell'Africa del Nord e dall'Asia Minore fino all'Estremo Oriente. Presente nella Regione Neartica, è stata diffusa in molte regioni come parassita dei cereali. Ampiamente presente in tutta Italia (Baldizzone et al., 2013). 
BIologiA: La larva vive normalmente in diversi funghi arboricoli.

Opogona omoscopa (Meyrick, 1893) [det. Gaedike] (Fig. 2)

Foce della Fiumara Amendolea, 9.VII.2013 (lux). Distribuzione GeograficA: Specie diffusa in Australia, Asia sudorientale, Regione Etiopica, isole dell'Oceano Indiano, è stata introdotta negli Stati Uniti e in Europa, dove è segnalata di Azzorre, Madera, Gibilterra, Spagna, Gran Bretagna, Danimarca, Francia, Corsica, Sardegna. Prima segnalazione per la parte continentale dell'Italia.

BIologia: La larva si sviluppa su vegetali in decomposizione, ma anche su piante vive, cui può arrecare danni.

Wegneria panchalcella (Staudinger, 1871) [det. Nel] Foce della Fiumara Amendolea, 9.VII.2013 (lux).

DistribuZIONE GEOGRAFICA: Ungheria, Grecia, Russia meridionale, Isole Canarie, Algeria, Regioni Afrotropicale, Orientale e Australiana (Tokár \& Buschmann, 2012). Seconda segnalazione per l'Italia. In precedenza un esemplare era stato raccolto in Basilicata da Bassi e Scaramozzino nel 1986 a Tursi (MT) (Bassi $\&$ Nel, 2015).

Biologia: Sconosciuta.

\section{Bucculatricidae [det. Triberti]}

Bucculatrix bechsteinella (Bechstein \& Scharfenberg, 1805)

Monte Ulis, 8.VII.2013 (lux).

DistribuZione GeOGRafica: Presente quasi ovunque in Europa (Karsholt \& Nieukerken, 2017). In Italia è molto diffusa e comune in tutta la penisola (Baldizzone et al., 2013).

Biologia: La larva mina le foglie di numerose Rosaceae: Crataegus spp., Malus spp., Pyrus spp., Sorbus spp.

Bucculatrix ulmella Zeller, 1848

Monte Ulis, 11.VII.2013 (lux).

DistribuZione GeOgraficA: Presente in tutta Europa e diffusa in tutta Italia, isole comprese (Baldizzone et al., 2013).

BiologiA: La larva mina le foglie di Fagus sylvatica, Castanea sativa e Quercus spp.

\section{Gracillariidae}

Calybites phasianipennella (Hübner, 1813)

Foce della Fiumara Amendolea, 9.VII.2013 (lux). DistRIBUZIONE GEOGRAFICA: Diffusa nella Regione Paleartica, ma presente anche in India e Cina meridionale (De Prins \& De Prins, 2018). In Italia presente ovunque (Gaedike et al., 1995).

BIOLOGIA: La specie è polifaga e preferibilmente vive su Polygonum spp., Rumex spp. e Lysimachia spp. È segnalata anche su varie specie dei generi Chenopodium, Symphytum, Hypericum, Lythrum e Oxyria. Frequenta soprattutto boschi umidi e paludi ma anche biotopi più asciutti come margini di coltivi (Triberti et al., 2017).

Acrocercops brongniardella (Fabricius, 1798)

Monte Ulis, 8.VII.2013 (lux).

DisTRIBUZIONE GEOGRAFICA: Non rara ma piuttosto localizzata, presente nell'intera Regione Paleartica (De Prins \& De Prins, 2018). In Italia segnalata ovunque (Gaedike et al., 1995).

BIologia: La larva mina le foglie di Quercus spp., incluso le querce sempreverdi. Predilige i boschi aperti, specialmente dove vi sono giovani alberi e le larve, nella fase finale della mina, sono spesso gregarie (Emmet et al., 1985).

Dextellia dorsilineella (Amsel, 1935) [det. Triberti] Foce Fiumara Amendolea, 9.VII.2013 (lux).

DistribuZIONE GEOGRAFICA: Specie molto rara, con distribuzione mediterranea (inclusa l'Africa settentrionale), ma recentemente trovata anche in Turkmenistan e Cina (De Prins \& De Prins, 2018). In Italia è nota solamente per le Marche e la Sardegna (Braggio \& Triberti, 2011). Prima segnalazione per l'Italia meridionale.

BIOLOGIA: La pianta ospite di questa specie è sconosciuta. Gli adulti sono sempre stati raccolti al volo o al lume in zone paludose (Triberti, com. pers.).

Phyllonorycter parisiella (Wocke, 1848) [det. Triberti]

Monte Ulis, 11.VII.2013 (lux).

DistribuZione GeOGRAFICA: Europa centrale (De Prins \& De Prins, 2018). In Italia è piuttosto rara e localizzata e non è conosciuta della Sicilia (Braggio \& Triberti, 2011).

BIOLOGIA: La larva vive a spese di Quercus pubescens 


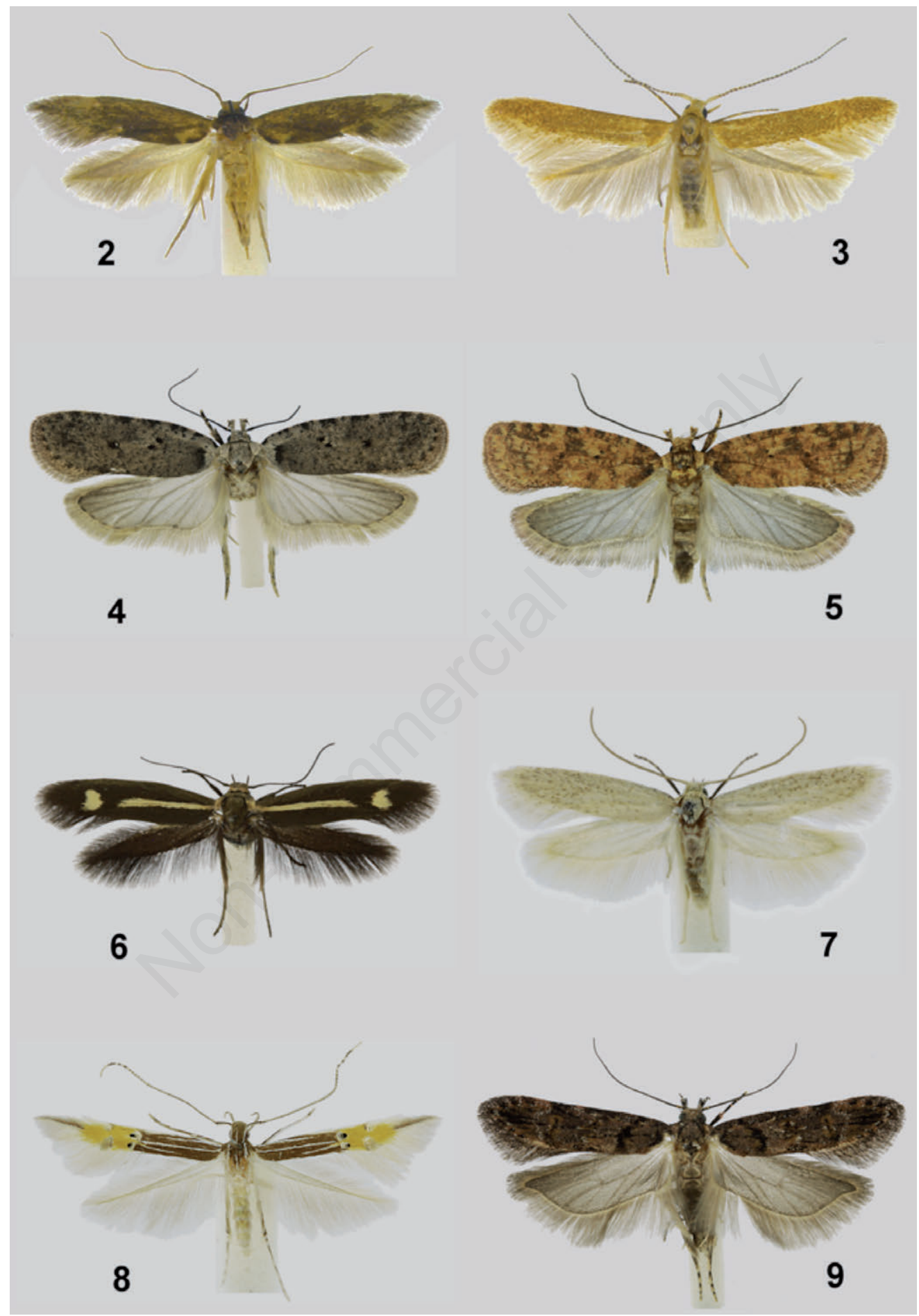

Figg. 2-9. Foto di alcune delle specie di maggiore interesse. 2 - Opogona omoscopa (Meyrick, 1893), (14 mm); 3 - Coleophora aspromontis Baldizzone, 2014 - $\widehat{O}$ (11 mm); 4 - Agonopterix thapsiella (Zeller, 1847), $\bigcirc$ (25 mm); 5 - Agonopterix ferocella

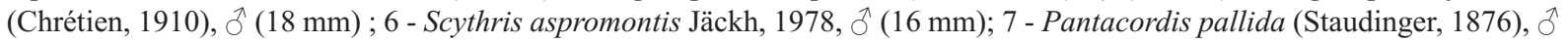
(12 mm); 8 - Cosmopterix coryphaea Walsingham, 1907, o (9 mm); 9 - Teleiopsis laetitiae Schmid, 2011, ổ (19 mm). 
ma talvolta è segnalata anche su $Q$. robur, Q. cerris e Q. ilex. Predilige boschi aperti in ambienti termofili (Triberti, com. pers.).

Phyllonorycter salicicolella (Sircom, 1848) [det. Triberti]

Monte Ulis, 8.VII.2013 (lux).

Distribuzione Geografica: Presente nella Regione Paleartica (De Prins \& De Prins, 2018), in Italia è segnalata solo per la parte settentrionale (Gaedike et al. 1995). Prima segnalazione per l'Italia meridionale.

Biologia: La larva è minatrice delle foglie di Salix spp., con rare segnalazioni su Populus spp.

Phyllonorycter maestingella (Müller, 1764) [det. Triberti]

Passo Praca, 6.VII.2013 (lux).

DISTRIBUZIONE GEOGRAFICA: Europa centro-settentrionale. In Italia è conosciuta della parte continentale ovunque ci sia il faggio (Baldizzone et al., 2013).

Biologia: La larva vive su foglie di Fagus sylvatica.

Phyllonorycter staintoniella (Nicelli, 1853) [det. Triberti] Monte Ulis, 8.VII.2013 (lux).

DISTRIBUZIONE GEOGRAFICA: La specie è presente in maniera molto discontinua in Europa ed è sempre piuttosto rara e localizzata (De Prins \& De Prins, 2018). In Italia è conosciuta delle regioni settentrionali e della Sardegna (Braggio \& Triberti, 2011). È stata utilizzata in Nuova Zelanda, Australia e alcuni stati degli USA nella lotta biologica contro il Cytisus scoparius. Prima segnalazione per l'Italia meridionale.

Biologia: La larva mina le foglie di Cytisus spp., Genista spp., Laburnum anagyroides, Lembotropis nigricans (Braggio \& Triberti, 2011).

Triberta cistifoliella (Groschke, 1944) [det. Triberti] Monte Ulis, 11.VII.2013 (lux).

DistRIBUZIONE GEOGRAFICA: T. cistifoliella è stata recentemente riportata al rango di specie valida (De Prins et al., 2013) ma la sua distribuzione è ancora incerta per la stretta somiglianza con Phyllonorycter helianthemella (Herrich-Schaffer, 1861), di cui era ritenuta sinonimo. Sinora sicuramente nota per le isole Canarie, Spagna, Macedonia, Sicilia, Grecia, Cipro. Prima segnalazione per l'Italia continentale.

Biologia: La larva mina le foglie di Cistus spp. ed è rinvenuta esclusivamente nelle zone xerotermiche (De Prins et al., 2013).

\section{Yponomeutidae}

Yponomeuta padella (Linnaeus, 1758)

Monte Ulis, 10.VII.2013 (lux).

DistribuZione GeOgrafica: Presente in gran parte dell'Europa. In Italia è conosciuta del territorio continentale e della Sardegna (Baldizzone et al., 2013).

Biologia: Larva su Rosaceae dei generi Cotoneaster, Crataegus, Malus, Pyrus, Prunus, in una tela di seta.

Zelleria hepariella Stainton, 1849

Monte Ulis, 8.VII.2013 (lux).

DistribuZIONE GEOGRAFICA: Diffusa in gran parte dell'Europa e in Asia Minore. Per l'Italia è segnalata del Nord e di Sicilia e Sardegna. Prima segnalazione per l'Italia meridionale.

BIOLOGIA: La larva si sviluppa su piante del genere Fraxinus, Phyllirea, Lonicera, ecc.

Cedestis subfasciella (Stephens, 1834)

Gambarie, 6.VII.2013 (lux).

DistribuZIONE GEOGRAFICA: Conosciuta di numerosi paesi d'Europa, in Italia, citata della parte settentrionale (Baraniak et al., 1995) è stata raccolta anche nel Lazio (Pinzari et al., 2010). Segnalata in l'Italia meridionale solo per la Sila (Scalercio et al., 2015).

Biologia: La larva si sviluppa su Pinus sylvestris e Abies pectinata, come minatrice degli aghi a partire dalla punta.

\section{Argyresthia pruniella (Clerck, 1759)}

Cucullaro, 12.VII.2013 (lux).

DistribuZIONE GEOGRAFICA: Specie comune in tutta Europa, diffusa fino all'Asia centrale. In Italia Baraniak et al. (1995) la citano di Nord, Sicilia e Sardegna, ma probabilmente è comune in tutte le regioni. Segnalata recentemente in Italia meridionale per la Sila (Scalercio et al., 2015).

Biologia: La larva attacca i fiori e i giovani frutti di alberi di varie specie (Cerasus spp., Corylus avellana, Crataegus spp., Malus spp., Prunus spp., Sorbus spp.).

\section{Bedelliidae}

Bedellia somnulentella (Zeller, 1847)

Monte Ulis, 7-8, 11.VII.2013 (lux); Foce della Fiumara Amendolea, 9.VII.2013 (lux)

DistribuZIONE GEOGRAFICA: Specie cosmopolita, rinvenuta anche in Oceania, tuttavia nell'Europa setten- 
trionale non si estende oltre la Danimarca e la Svezia meridionale. In Italia è presente ovunque (Baldizzone et al., 2013).

BIologia: La larva vive sulle Convolvulaceae, particolarmente sulle specie dei generi Calystegia, Convolvulus, Ipomoea.

\section{Ypsolophidae}

\section{Ypsolopha parenthesella (Linnaeus, 1761)}

Gambarie, 6.VII.2013 (lux).

Distribuzione Geografica: Europa, Asia Minore, Asia fino alla Cina. In Italia è citata per tutta la penisola, ma non si hanno dati per le isole (Baraniak et al., 1995).

Biologia: Larva su piante arboree di vari generi, quali Quercus, Fagus, Fraxinus, Populus, Carpinus, Crataegus, Malus, Betula e Corylus avellana.

Ypsolopha ustella (Clerck, 1759)

Cucullaro, 12.VII.2013 (lux).

DisTRIBUZIONE GEOGRAFICA: Europa settentrionale e centrale fino all'Asia Minore. In Italia è citata per tutta la penisola, ma non si hanno dati per le isole (Baldizzone et al., 2013).

BIOLOGIA: Larva su Acer campestre, segnalata anche di A. pseudoplatanus.

\section{Acrolepiidae}

Acrolepiopsis vesperella (Zeller, 1850)

Vallone delle Serre, 7.VII.2013 (lux).

DistribuZione GeOGRAFICA: Conosciuta di Portogallo, Spagna, Francia, Germania, Italia, Croazia, Serbia, Montenegro, Isole Canarie. Per l'Italia è conosciuta di tutto il territorio continentale, Sicilia e Sardegna.

Biologia: Larva su piante dei generi Smilax e Tamus.

\section{Ethmiidae}

Ethmia bipunctella (Fabricius, 1775)

Monte Ulis, 11.VII.2013 (lux).

DistribuZIONE GeOgRAFICA: Diffusa, ma a volte localizzata in Europa centrale e meridionale, Isole Canarie, Africa del Nord, Medio Oriente fino all'Iran. Introdotta accidentalmente in Canada e USA. In Italia è segnalata di tutto il territorio, isole comprese (Baldizzone et al., 2013).

Biologia: Larva su Echium vulgare e Symphytum spp.

\section{Depressariidae [det. Nel]}

Agonopterix thapsiella (Zeller, 1847) (Fig. 4)

Monte Ulis, 11.VII.2013 (lux).

Distribuzione geografica: Conosciuta della Penisola Iberica, Francia, Italia, Ungheria, Malta, Grecia, Creta, Vicino Oriente, Africa del Nord. In Italia è segnalata per il Nord, Lazio, Sicilia e Sardegna (Baldizzone et al., 2013). Prima segnalazione per l'Italia meridionale. Biologia: La larva vive su varie ombrellifere, quali Ferula communis, Foeniculum vulgare, Heracleum spp., Scandix spp., Seseli tortuosum, Thapsia villosa.

Agonopterix ferocella (Chrétien, 1910) (Fig. 5)

Monte Ulis, 8 e 10.VII.2013 (lux)

DisTRIBUZIONE GEOGRAFICA: Francia meridionale, Italia, Ucraina, Russia. Segnalata solo per l'Italia settentrionale (Baldizzone et al., 2013). Prima segnalazione per l'Italia centro-meridionale.

Biologia: Larva su Cirsium ferox e Echinops sp.

Agonopterix nodiflorella (Millière, 1866)

Monte Ulis, 8 e 10.VII.2013 (lux).

DistribuZIONE GEOGRAFICA: Specie prevalentemente mediterranea, conosciuta della Penisola Iberica, Isole Canarie, Francia, Italia, Macedonia, Ucraina, Cipro, Vicino Oriente, Africa del Nord. In Italia è citata del territorio continentale, di Sicilia e Sardegna (Baldizzone et al., 2013).

Biologia: La larva vive su Ferula communis.

Agonopterix scopariella (Heinemann, 1870)

Monte Ulis, 8.VII.2013 (lux).

Distribuzione geografica: Presente in larga parte dell'Europa, tranne la parte settentrionale, dove arriva solo alla Norvegia del Sud, Vicino Oriente, Africa del Nord. In Italia è citata per il Nord, Lazio, Sicilia e Sardegna (Baldizzone et al., 2013). Prima segnalazione per l'Italia meridionale.

Biologia: Larva su Cytisus scoparius e Lupinus arboreus.

Agonopterix nervosa (Haworth, 1811)

Monte Ulis, 8.VII.2013 (lux).

Distribuzione Geografica: Tutta l'Europa, Asia Minore, USA e Canada. In Italia è citata delle regioni settentrionali, Sicilia e Sardegna (Baldizzone et al., 2013). Prima segnalazione per l'Italia centro-meridionale. 
Biologia: Larva oligofaga su diverse leguminose arbustive (Baldizzone et al., 2013).

\section{Elachistidae [det. Varalda]}

Elachista dispunctella (Duponchel, 1843)

Monte Ulis, 8-11.VII.2013 (lux).

DistRIBUZIONE GEOGRAFICA: Inghilterra, Europa centrale e meridionale ad eccezione della Penisola Iberica e dall'Europa orientale si spinge fino al Kazakistan; è presente anche in Turchia. In Italia era nota solo per il Nord (Baldizzone et al., 2013), per cui questa è la prima segnalazione per la parte centro-meridionale. Nella pubblicazione di Kaila (2015) sul gruppo di Elachista dispunctella, la specie non è citata per l'Italia, ma gli esemplari raccolti in Calabria confermano la presenza della specie nel nostro Paese (Varalda, com. pers.).

Biologia: Larva su Poaceae: Festuca sp., ? Festuca ovina, Festuca rubra.

Elachista subocellea (Stephens, 1834)

Monte Ulis, 8.VII.2013 (lux).

DistribuZione GeOgRAFICA: Quasi tutta Europa. In Italia è presente in tutta la penisola (Baldizzone et al., 2013).

BIOLOGIA: Larva su Poaceae: Brachypodium pinnatum e B. sylvaticum.

\section{Scythrididae}

\section{Scythris aspromontis Jäckh, 1978 (Fig. 6)}

Cucullaro, 11.VII.2013.

DistribuZIONE GEOGRAFICA: Conosciuta solo di poche località della Francia meridionale e dell'Italia centrale e meridionale.

Biologia: La specie è stata allevata da Thierry Varenne su una Cariofillacea non ancora identificata (Nel, com. epist.).

\section{Oecophoridae}

Metalampra italica Baldizzone, 1977

Cucullaro, 11.VII.2013.

DistribuZIONE GEOGRAFICA: Ritenuta un endemismo italiano per molti anni, è stata poi scoperta anche in Svizzera, Germania e Gran Bretagna; in Italia è diffusa dalle regioni settentrionali fino al Sud della penisola e non è conosciuta per le isole (Baldizzone et al., 2013). Segnalata recentemente in Calabria per Sila e Catena Costiera (Leonetti et al., in stampa).
Biologia: La larva si sviluppa nel legno secco di varie piante tra cui Ostrya carpinifolia.

Kasyniana diminutella (Rebel, 1931)

Gambarie, 6.VII.2013 (lux); Monte Ulis, 10, 11.VII.2013 (lux); Cucullaro, 12.VII.2013 (lux).

Distribuzione Geografica: Conosciuta di parte dell'Europa centrale (Austria, Slovacchia, Ungheria, Romania) e di quella meridionale (Francia, Italia, Sicilia, Slovenia e Croazia); presente anche in Asia Minore e Marocco. Per l'Italia è nota di alcune regioni della parte continentale e della Sicilia (Baldizzone et al., 2013). Segnalata recentemente in Calabria per la Catena Costiera (Leonetti et al., in stampa).

Biologia: Sconosciuta.

Herrichia excelsella Staudinger, 1871

Gambarie, 6.VII.2013 (lux); Vallone delle Serre, 7.VII.2013 (lux); Monte Ulis, 7.VII.2013 (lux).

DisTRIBUZIONE GEOGRAFICA: Specie localizzata, conosciuta di poche zone dell'Europa centrale (Austria, Svizzera, Germania) e di quella meridionale (Penisola Iberica, Francia, Italia e Sicilia) (Tokár et al., 2005). In Italia è conosciuta di Valle d'Aosta, Piemonte, Trentino-Alto Adige e Lazio (Baldizzone et al., 2013). Segnalata recentemente in Italia meridionale per Sila (Scalercio et al., 2015) e Serre Vibonesi (Leonetti et al., in stampa).

Biologia: Non conosciuta, ma si pensa che la larva viva nel legno morto delle cortecce di Pinus spp. e di altre conifere.

Batia lambdella (Donovan, 1793)

Cucullaro, 12.VII.2013 (lux).

DISTRIBUZIONE GEOGRAFICA: Europa centrale, occidentale e meridionale fino all'Inghilterra (manca in Scandinavia), Palestina, Africa settentrionale. Conosciuta di tutta Italia, isole maggiori comprese (Baldizzone et al., 2013).

Biologia: Larva nel legno morto di Ulex e di alcune specie arboree (Aesculus spp., Alnus spp., Carpinus spp.).

Batia lunaris (Haworth, 1828)

Monte Ulis, 10.VII.2013 (lux); Cucullaro, 12.VII.2013 (lux).

DistribuZione Geografica: Presente in larga parte dell'Europa, dalla Gran Bretagna alla Penisola Iberica fino alla Russia, ma non in Scandinavia; conosciuta 
anche di Asia Minore e Africa del Nord e introdotta accidentalmente in America settentrionale. Segnalata di tutta Italia, isole maggiori comprese (Baldizzone et al., 2013).

BIologia: Larva nei funghi e licheni presenti su vecchi alberi o nel legno di alberi morenti, sotto la corteccia.

Dasycera oliviella (Fabricius, 1794)

Gambarie, 6.VII.2013 (lux).

DistribuZione GeOgRAFICA: Presente in Gran Bretagna e nella maggior parte dell'Europa centrale e meridionale, fino all'Ucraina e alla Turchia. In Italia è conosciuta della parte continentale e della Sicilia (Baldizzone et al., 2013).

BIOLOGIA: Larva sotto la corteccia di alberi morti.

\section{Lecithoceridae}

Lecithocera nigrana (Duponchel, 1836)

Monte Ulis, 10-11.VII.2013 (lux).

DisTRIBUZIONE GEOGRAFICA: Dalla Spagna all'Ucraina e all'Anatolia. In Italia si hanno dati per Valle d'Aosta, Piemonte, Lombardia, Veneto, Trentino-Alto Adige, Liguria, Toscana, Abruzzo, Lazio, Sicilia (Baldizzone et al., 2013). Prima segnalazione per l'Italia meridionale.

BiologiA: Larva detritofaga nella lettiera.

Odites kollarella (O. Costa, 1832)

Monte Ulis, 8.VII.2013 (lux).

DistribuZIONE GEOGRAFICA: Dalla Spagna all'Ucraina e all'Anatolia. In Italia si hanno dati per Valle d'Aosta, Piemonte, Lombardia, Veneto, Trentino-Alto Adige, Liguria, Toscana, Abruzzo, Lazio, Sicilia (Baldizzone et al., 2013). Prima segnalazione per l'Italia meridionale

BIOLOGIA: Larva detritofaga nella lettiera.

\section{Coleophoridae}

Coleophora aspromontis Baldizzone, 2014 (Fig. 3) Monte Ulis, 8, 10-11.VII.2013 (lux). Tutti gli esemplari raccolti sono stati inseriti nella descrizione della specie, holotypus e paratypi.

DistRIBUZIONE GEOGRAFICA: Specie di recente descrizione probabilmente endemica dell'Aspromonte.

Biologia: La larva si sviluppa su Quercus pubescens (Baldizzone, 2018).
Coleophora flavipennella (Duponchel, 1843)

Monte Ulis, 10.VII.2013 (lux).

DistribuZIone Geografica: Tutta l'Europa, Turchia, Caucaso, Russia (Urali merid.), Tunisia. In Italia è conosciuta di tutta la penisola, dal Nord fino alla Calabria, ma non si hanno dati per le isole.

Biologia: Monovoltina, dalla fine di giugno e in luglio. Larva normalmente sulle querce a foglia caduca con una complessa biologia biennale, che comporta la costruzione di vari astucci di foggia differente.

Coleophora alnifoliae Barasch, 1934

Cucullaro, 12.VII.2013 (lux).

Distribuzione GEOGRAFICA: Quasi tutta l'Europa, Caucaso, Siberia centrale, Cina, Canada, USA. In Italia si hanno dati per Piemonte, Trentino-Alto Adige, Fiuli-Venezia Giulia e Sardegna. Prima segnalazione per l'Italia centro-meridionale.

Biologia: La larva si sviluppa su piante del genere Alnus, come minatrice fogliare, dentro ad un astuccio larvale costruito con un lembo ritagliato dal bordo di una foglia.

Coleophora ptarmicia Walsingham, 1910

Monte Ulis, 9-10.VII.2013 (lux).

DistribuZione Geografica: Paesi Baltici, Francia, Italia, Sicilia, Austria, Croazia, Rep. Ceca, Slovacchia, Ungheria, Bulgaria, Grecia, Ucraina, Russia (Basso Volga), Turchia, Altai, Cina. Specie termofila, per l'Italia è conosciuta solo di una zona xerotermica delle Alpi Marittime (Piemonte) e di parecchie località della Sicilia. Prima segnalazione per l'Italia centro-meridionale.

BIOLOGIA: Piante nutrici Achillea di varie specie (millefolium, ptarmica, setacea).

\section{Coleophora hemerobiella (Scopoli, 1763)}

Monte Ulis, 10.VII.2013 (lux).

DistribuZIONE GeOgrafica: Quasi tutta l'Europa, Turchia, Armenia, Russia (Urali meridionali), Turkmenistan, Siria, Iran. In Italia è presente in tutta la penisola, Sicilia e Sardegna. Prima segnalazione per la Calabria. Biologia: Monovoltina, con schiusure a fine giugno e luglio. La larva vive su numerose specie arboree e arbustive di Rosaceae.

Coleophora mayrella (Hübner, 1813)

Gambarie, 6.VII.2013 (lux); Vallone delle Serre, 7.VII.2013 (lux); Cucullaro, 12.VII.2013 (lux). 
DisTRIBUZIONE GEOGRAFICA: Europa, Africa settentrionale, Anatolia, Libano, Siria, Iran, America settentrionale, Cile. Conosciuta di tutta Italia, isole comprese. BIologia: Monovoltina, presente a maggio e soprattutto in giugno, ma talvolta anche in luglio alle quote più alte. Larva nei fiori del trifoglio bianco (Trifolium repens), ma probabilmente anche in altre specie del genere Trifolium.

\section{Coleophora zelleriella Heinemann, 1854}

Gambarie, 6.VII.2013 (lux); Vallone delle Serre, 7.VII.2013 (lux); Monte Ulis, 10.VII.2013 (lux)

DistribuZIOne GeOgRAFICA: Quasi tutta Europa (manca in Gran Bretagna), Anatolia, Siria, Iran. In Italia si hanno dati per diverse regioni, dal Nord fino alla Basilicata, mentre la specie non è conosciuta per le isole. Prima segnalazione per la Calabria.

BIOLOGIA: Monovoltina, con schiusure che si protraggono per tutto giugno e parte di luglio. Larva in un astuccio di seta nera su Quercus spp. e Salix spp.

\section{Coleophora pulchripennella Baldizzone, 2011}

Monte Ulis, 10.VII.2013 (lux).

DistRIBUZIONE GEOGRAFICA: La specie è largamente diffusa in tutta la regione mediterranea, dalla Francia, dove risale fino alla costa atlantica nella Gironde, alla Penisola Iberica, all'Italia, alla Croazia (Dalmazia) fino alla Grecia (compresa Creta e varie isole minori), e alla Turchia; è conosciuta anche di Malta e della Tunisia. In Italia si hanno numerosi dati, a partire dal Piemonte meridionale (Capanne di Marcarolo), che per ora è il limite settentrionale di questa specie nel territorio italiano; la si conosce di Liguria, Lazio, Umbria, Campania, Sicilia e Sardegna, ma probabilmente è molto più diffusa. Prima segnalazione per la Calabria. BIOLOGIA: Monovoltina nelle zone più settentrionali, con schiusure da metà giugno a fine luglio. Larva su giovani foglie apicali e sui fiori di Erica carnea in un astuccio allungato, costruito con seta nera.

\section{Coleophora helichrysiella Krone, 1909}

Monte Ulis, 10.VII.2013 (lux).

DistribuZione GEOGRAFICA: Europa meridionale, da Portogallo e Spagna, attraverso Francia, Corsica, Italia e Croazia, fino ai Balcani, Romania e Creta. In Italia è molto localizzata ed è conosciuta solo di poche località di alcune regioni continentali: Trentino-Alto Adige, Liguria, Lazio. Prima segnalazione per la Calabria.
Biologia: Le larve si alimentano di Helichrysum italicum e H. stoechas.

Coleophora alticolella Zeller, 1849

Gambarie, 6.VII.2013 (lux).

DistribuZione geograficA: Europa, Tunisia, Iran, Stati Uniti. In Italia è conosciuta di tutta la parte continentale e della Sardegna.

BiologiA: Monovoltina, presente in giugno e luglio. Larva si nutre di semi di Juncus di varie specie.

Coleophora taeniipennella Herrich-Schäffer, 1855 Foce della Fiumara Amendolea, 9.VII.2013 (lux). DisTRIBUZIONE GEOGRAFICA: Quasi tutta l'Europa, Anatolia, Caucaso, Armenia, Iran. In Italia è diffusa dal Nord fino alla Calabria ed è conosciuta anche per la Sardegna. BIologia: Monovoltina, presente in giugno e luglio. Larva su Juncus di varie specie. Come per la specie precedente, si nutre di semi in un piccolo astuccio cilindrico, di color bianco sporco, costruito con seta.

Coleophora lassella Staudinger, 1859

Foce della Fiumara Amendolea, 9.VII.2013 (lux). DistribuZione GEOGRAFICA: Isole Canarie, Portogallo, Spagna, Francia, Italia, Sicilia, Sardegna, Croazia, Polonia, Romania, Malta, Grecia, Cipro, Marocco, Russia (Basso Volga), Turchia, Caucaso, Iran, Afghanistan. In Italia è specie localizzata, conosciuta solo del Piemonte meridionale, per quanto riguarda il Nord, mentre si hanno dati per diverse regioni centrali e meridionali, oltre che per le isole: Toscana, Marche, Lazio, Puglia, Sicilia, Sardegna. Prima segnalazione per la Calabria. Bilogia: Larva su Juncus bufonius.

\section{Blastobasidae}

Blastobasis phycidella (Zeller, 1839) Gambarie, 6.VII.2013 (lux); Monte Ulis, 11.VII.2013 (lux).

DistribuZIONE GEOGRAFICA: Quasi tutta l'Europa, a parte la Scandinavia. In Italia è presente in tutto il territorio, isole maggiori comprese (Baldizzone et al., 2013). BIologia: Larva nel legno in decomposizione, in particolare di querce.

Autostichidae [det. Nel]

Oegoconia deauratella (Herrich-Schäffer, 1854)

Monte Ulis, 10.VII.2013 (lux) 
DisTRIBUZIONE GEOGRAFICA: Gran Bretagna, parte dell'Europa centrale e meridionale, non presente nei Paesi Baltici; la sua distribuzione è conosciuta in modo non ancora soddisfacente. In Italia è citata per il territorio continentale (Baldizzone et al., 2013).

BIOLOGIA: La larva vive nella lettiera.

Pantacordis pallida (Staudinger, 1876) (Fig. 7)

Monte Ulis, 7-11.VII.2013 (lux).

DistribuZione GeograficA: Specie localizzata conosciuta solo di Puglia, Sicilia, Malta, Libia, Tunisia, Algeria e Marocco. Prima segnalazione per la Calabria. BIOLOGIA: La biologia è sconosciuta (Gozmány, 2008).

\section{Amphisbatidae}

Pseudatemelia subochreella (Doubleday, 1859)

Gambarie, 6.VII.2013 (lux).

DistRIBUZIONE GEOGRAFICA: Dalla Gran Bretagna alla Svezia meridionale a Nord, Europa centrale e meridionale fino al Caucaso a Est e al Marocco a Sud. In Italia è conosciuta del territorio continentale e della Sicilia (Baldizzone et al., 2013).

Biologia: La larva si costruisce un astuccio con una foglia ripiegata di Betula spp., Carpinus betulus, Fagus sylvatica, Quercus spp. e si nutre di foglie in decomposizione nella lettiera.

\section{Momphidae}

Mompha epilobiella (Denis \& Schiffermüller, 1775) Foce della Fiumara Amendolea, 9.VII.2013 (lux). Distribuzione GEOGRAFicA: Europa, Asia Minore, Caucaso, regioni submontane dell'Asia Centrale, America del Nord (probabilmente introdotta) (Koster $\&$ Sinev, 2003). In Italia è segnalata per la parte continentale (Riedl, 1995) e anche per la Sicilia (Karsholt \& Nieukerken, 2017).

Biologia: Larva su Epilobium hirsutum e più raramente su altre specie del genere Epilobium (Koster \& Sinev, 2003).

\section{Cosmopterigidae [det. Koster]}

Cosmopterix coryphaea Walsingham, 1908 (Fig. 8) Foce della Fiumara Amendolea, 9.VII.2013 (lux). DistribUZIONE GEOGRAFICA: Europa meridionale, Africa settentrionale, Vicino Oriente, Canarie. In Italia Riedl (1995) la cita delle regioni settentrionali e della
Sicilia. Prima segnalazione per l'Italia meridionale. Biologia: La larva mina le foglie di Phragmites australis (Koster \& Sinev, 2003).

Eteobalea intermediella (Riedl, 1966)

Monte Ulis, 11.VII.2013 (lux).

DistribuZIONE GEOGRAFICA: Europa centrale e meridionale, Caucaso, Asia Minore, Iran, Asia Centrale (Koster \& Sinev, 2003). In Italia è citata di tutto il territorio, senza indicazioni precise relative alle regioni, isole maggiori comprese (Riedl, 1995). Prima segnalazione per la Calabria.

BIOLOGIA: Larva su varie specie del genere Linaria.

Ascalenia vanella (Frey, 1860)

Foce della Fiumara Amendolea, 9.VII.2013 (lux). DistRIBUZIONE GEOGRAFICA: Europa centrale e meridionale, Canarie, Asia Minore, Caucaso (Koster \& Sinev, 2003). In Italia è segnalata della parte continentale, senza indicazioni delle regioni e della Sicilia (Karsholt \& Nieukerken, 2017). Prima segnalazione per la Calabria.

Biologia: Larva su Myricaria germanica e Tamarix sp. (Koster \& Sinev, 2003).

\section{Gelechiidae}

Isophrictis anthemidella (Wocke, 1871) [det. Nel] Monte Ulis 7.VII.2013 (lux).

DistribuZione GEOGRAFICA: Largamente diffusa in Europa, dal Portogallo, Spagna e Francia fino alla Finlandia in direzione Nord e all'Asia centrale in direzione Est. Presente in tutta Italia, Sicilia e Sardegna comprese (Huemer \& Karsholt, 1995).

Biologia: Larva segnalata su Achillea ptarmica, Anthemis tinctoria, Pyrethrum corymbosum.

Metzneria aestivella (Zeller, 1839) [det. Karsholt] Monte Ulis, 7.VII.2013 (lux).

DisTRIBUZIONE GEOGRAFICA: Largamente diffusa in buona parte dell'Europa, Isole Canarie fino all'Asia Centrale (Elsner et al., 1999). Presente in tutta Italia, Sicilia e Sardegna comprese (Huemer \& Karsholt, 1995). Biologia: Larva su piante del genere Carlina e su Pyrethrum corymbosum.

Ptocheuusa paupella (Zeller, 1847) [det. Karsholt] Foce della Fiumara Amendolea, 9.VII.2013 (lux). DistribuZIONE GEOGRAFICA: Gran Bretagna, Belgio, 
Olanda, Austria, Italia, Francia, Penisola Iberica, Croazia, Macedonia, Grecia. In Italia è largamente diffusa, e conosciuta anche di Sicilia e Sardegna (Baldizzone et al., 2013).

Biologia: Larva su Inula conyza, I. montana, Pulicaria dysenterica.

Monochroa cytisella (Curtis, 1837) [det. Karsholt] Monte Ulis, 7.VII.2013 (lux).

DistribuZIONE GEOGRAFICA: Largamente diffusa in tutta la regione paleartica. In Italia è presente in tutta la parte continentale, in Sicilia e Sardegna (Baldizzone et al., 2013).

Biologia: Larva su Pteridium aquilinum.

Monochroa melagonella (Constant, 1895) [det. Karsholt]

Monte Ulis, 8.VII.2013 (lux).

DistribUZIONE GeOGRAFICA: Specie conosciuta di poche località xeriche di Austria, Rep. Ceca, Slovacchia e Ungheria, oltre che della Penisola Iberica, Francia, Italia, Cipro. In Italia è segnalata solo per il Nord (Baldizzone et al., 2013). Prima segnalazione per l'Italia centro-meridionale.

Biologia: Larva su Rubia peregrina.

Eulamprotes nigromaculella (Millière, 1872) [det. Karsholt]

Monte Ulis, 7-8,10.VII.2013 (lux).

DistribuZione GEOGRAFICA: Portogallo, Spagna, Francia, Italia (comprese Sicilia e Sardegna), Grecia, Cipro, Ucraina (Karsholt \& Nieukerken, 2017). Prima citazione per la Calabria.

BIologia: La larva si sviluppa forse su Hypericum quadrangulum.

Ornativalva heluanensis (Debski, 1913) [det. Karsholt] (Fig. 11)

Foce della Fiumara Amendolea, 9.VII.2013 (lux).

DistribuZione GEOGRAFICA: Spagna, Croazia, Ucraina, Russia, Sicilia, Malta, Cipro, Isole Canarie, Africa settentrionale, Vicino e Medio Oriente fino al Pakistan e alla Mongolia (Karsholt \& Nieukerken, 2017). Nuova per la penisola italiana.

BIOLOGia: La larva si sviluppa su Frankenia spp. e Tamarix spp.

Ornativalva plutelliformis (Staudinger, 1859) [det. Karsholt]
Foce della Fiumara Amendolea, 9.VII.2013 (lux). DistribuZione geograficA: Penisola Iberica, Francia, Italia (comprese Sicilia e Sardegna), Romania, Ungheria, Macedonia, Grecia, Ucraina, Russia meridionale, Africa settentrionale, Asia centrale fino alla Cina. Biologia: Larva su piante del genere Tamarix.

Bryotropha senectella (Zeller, 1839) [det. Karsholt] Gambarie, 6.VII.2013 (lux).

Distribuzione geograficA: Diffusa in quasi tutta l'Europa eccetto la Penisola Iberica; presente anche in Cina. In Italia è conosciuta della parte continentale e della Sicilia (Baldizzone et al., 2013). Prima segnalazione per la Calabria.

BIOLOGIA: Le larve vivono a spese di varie specie di muschi.

Bryotropha dryadella (Zeller, 1850) [det. Karsholt] Monte Ulis, 8.VII.2013 (lux).

Distribuzione geografica: Gran Bretagna, Portogallo, Spagna, Francia, Corsica, Italia (comprese Sicilia e Sardegna), Albania, Macedonia, Bulgaria, Grecia, Creta, Algeria. Prima segnalazione per la Calabria.

BIOLOGIA: Le larve vivono a spese di varie specie di muschi.

Bryotropha italica Karsholt \& Rutten, 2005 [det. Karsholt]

Gambarie, 6.VII.2013 (lux).

DisTRIBUZIONE GEOGRAFICA: La specie è conosciuta solo dell'Italia, descritta in base ad esemplari raccolti da Baldizzone e Bassi sul Monte Pollino (versanti calabrese e lucano) ed è conosciuta di Calabria (presente anche in Sila) e dell'Abruzzo (Karsholt \& Rutten, 2005).

Biologia: Sconosciuta.

Recurvaria nanella (Denis \& Schiffermüller, 1775) [det. Karsholt]

Monte Ulis, 7.VII.2013 (lux).

DistRIBUZIONE GEOGRAFICA: Quasi tutta l'Europa fino all'Asia centrale; Nord Africa; America settentrionale (Canada e USA) dove forse è stata introdotta accidentalmente. Conosciuta di tutta Italia, isole comprese (Baldizzone et al., 2013).

BiologiA: La larva vive su varie piante arbustive e arboree, soprattutto della famiglia Rosaceae. Tra le principali sono segnalate Amelanchier ovalis, 
Crataegus spp., Cydonia spp., Malus spp., Prunus avium, P. cerasus, P. mahaleb, Pyrus communis, Sorbus spp.

Exoteleia dodecella (Linnaeus, 1758) [det. Nel] Gambarie, 6.VII.2013 (lux).

DistribuZione GEOGRAFICA: Regione paleartica, dall'Europa occidentale fino alla Siberia Sud-orientale; presente in Nord America. In Italia la specie è stata segnalata per la zona continentale (Baldizzone $e t$ al., 2013). Prima segnalazione per la Calabria.

Biologia: La specie è infeudata al Pinus sylvestris e ad altre specie dei generi Abies, Picea, Pinus. Le larve si trovano dall'autunno alla primavera, inizialmente come minatrici degli aghi, che successivamente uniscono insieme con una struttura sericea.

Teleiopsis diffinis (Haworth, 1828) [det. Karsholt] Monte Ulis, 7-8, 10.VII.2013 (lux).

DistRIBUZIONE GEOGRAFICA: Largamente diffusa in tutta Europa. In Italia è presente in tutto il territorio, comprese Sicilia e Sardegna (Baldizzone et al., 2013). Biologia: Larva su Acetosella vulgaris.

Teleiopsis laetitiae Schmid, 2011 [det. Karsholt] (Fig. 9)

Vallone delle Serre, 7.VII.2013 (lux).

Distribuzione Geografica: Svizzera, Austria, Italia, Macedonia, Grecia, Turchia. Per l'Italia si hanno dati per un esemplare del Trentino-Alto Adige e uno dell'Abruzzo (Schmid, 2011). La specie è nuova per l'Italia meridionale.

Biologia: Larva sconosciuta.

Pseudotelphusa paripunctella (Thunberg, 1794) [det. Karsholt]

Gambarie, 6.VII.2013 (lux).

DistribUZIONE GEOGRAFICA: Largamente diffusa in Europa, conosciuta anche di Cina e Giappone. In Italia è segnalata di tutta la penisola e della Sicilia (Baldizzone et al., 2013).

Biologia: Si sviluppa su Myrica gale, Betula spp., Fagus sylvatica, Quercus petraea, Hippophae rhamnoides.

Xenolechia aethiops (Humphreys \& Westwood, 1845) [det. Karsholt]

Gambarie, 6.VII.2013 (lux).

DistribuZIONE GEOGRAFICA: Specie localizzata. In Eu- ropa è conosciuta di Gran Bretagna, Olanda, Danimarca, Germania, Francia, e si estende verso Sud all'Italia e a Est all'Asia Minore, fino all'Estremo Oriente. In Italia è segnalata per il territorio continentale e la Sardegna (Baldizzone et al, 2013).

Biologia: Larva su Calluna vulgaris ed Erica spp.

Aroga velocella (Duponchel, 1838) [det. Karsholt] Monte Ulis, 7-8.VII.2013 (lux); Cucullaro, 12.VII.2013 (lux).

DistribuZione GeOgRAFICA: Largamente diffusa in Europa fino alla Turchia. In Italia è conosciuta di tutta la parte continentale e della Sardegna (Baldizzone et al., 2013).

Biologia: La larva si nutre delle foglie di Acetosella vulgaris.

Neophriseria peliella (Treitschke, 1835) [det. Karsholt]

Vallone delle Serre, 7.VII.2013 (lux).

DistRIBUZIONE GEOGRAFICA: Largamente diffusa in buona parte dell'Europa e presente in Africa settentrionale. In Italia è citata del territorio continentale e della Sardegna (Baldizzone et al., 2013). Prima segnalazione per la Calabria.

Biologia: Pianta alimentare è Acetosella vulgaris.

Neophriseria singula (Staudinger, 1876) [det. Karsholt] (Fig. 10)

Monte Ulis, 7.VII.2013 (lux).

DisTRIBUZIONE GEOGRAFICA: Buona parte dell'Europa, tranne Norvegia, Finlandia, Belgio, Svizzera, Balcani e Ucraina. Per l'Italia è citata solo di Sicilia e con dubbio per la Sardegna (Karsholt \& Nieukerken, 2017). Prima segnalazione per la penisola italiana.

BiologiA: Pianta alimentare è Acetosella vulgaris.

Scrobipalpa vasconiella (Rössler, 1877) [det. Karsholt]

Monte Ulis, 8.VII.2013 (lux).

DisTRIBUZIONE GEOGRAFICA: Distribuita in modo frammentario nella regione mediterranea, dalle Isole $\mathrm{Ca}$ narie a Madera alla Penisola Iberica, Francia, Italia, Grecia, Russia meridionale. Presente anche in Turchia, Africa settentrionale, Iran, Afghanistan. Per l'Italia è segnalata del territorio peninsulare e delle isole maggiori (Huemer \& Karsholt, 2010). Prima segnalazione per la Calabria.

Biologia: Sconosciuta. 


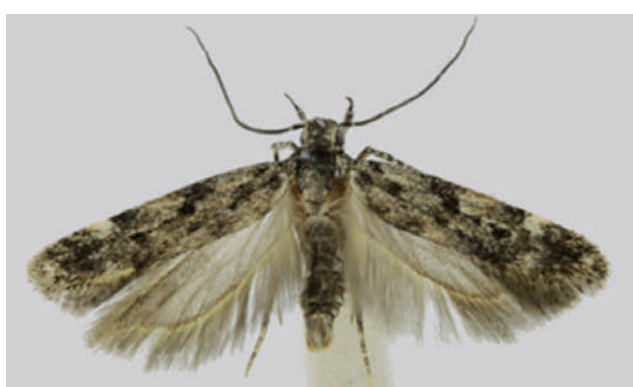

10

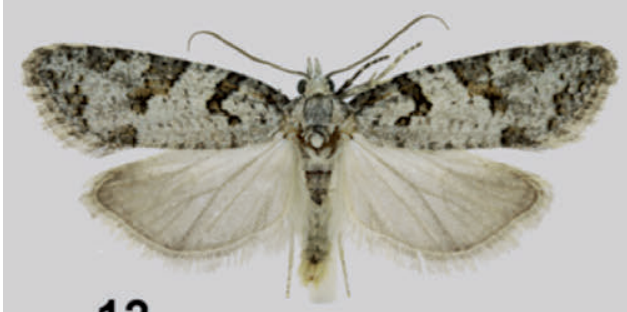

12

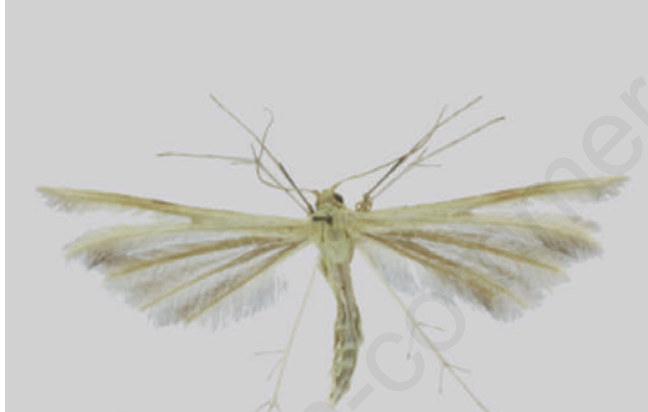

14

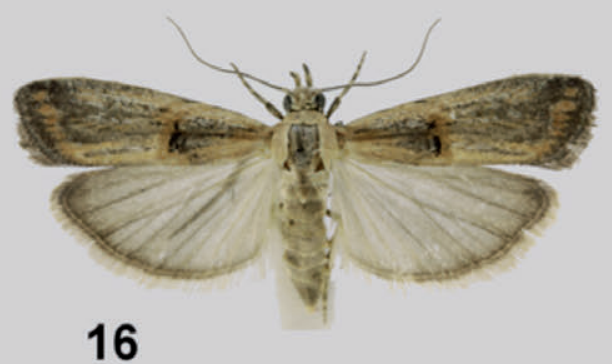

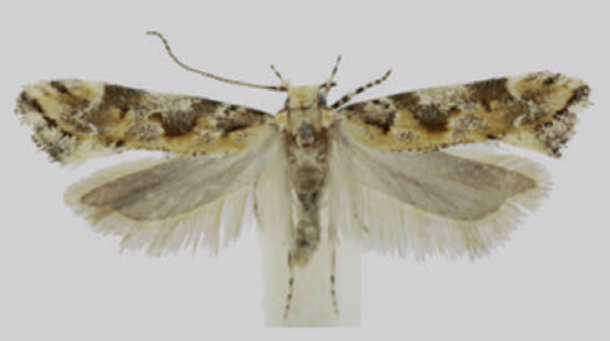

11

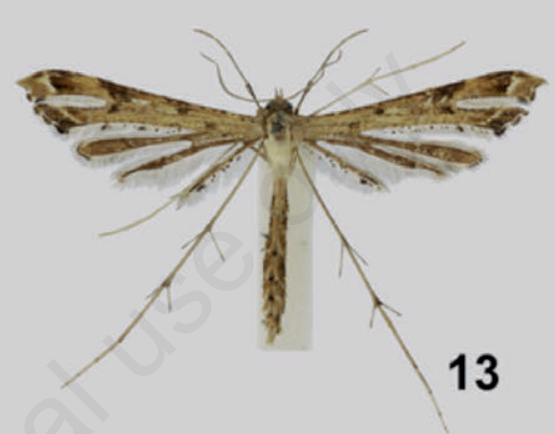

13

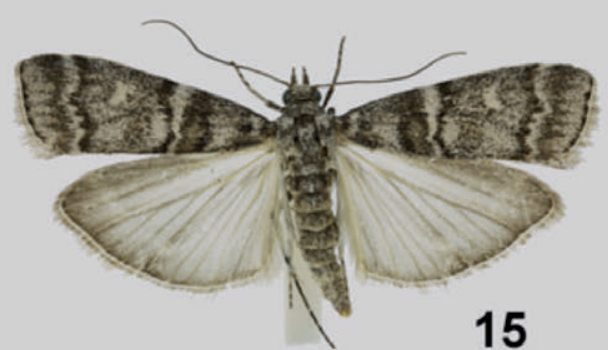

15

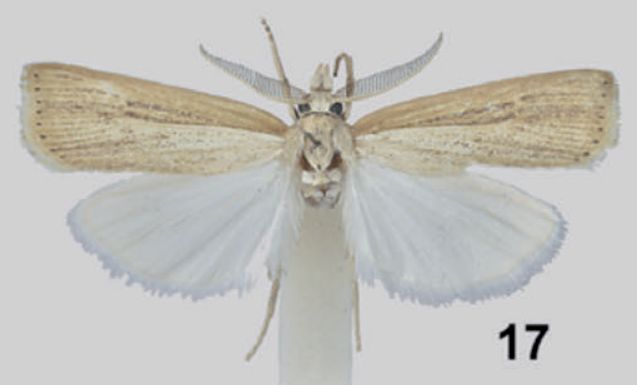

Figg. 10-17. Foto di alcune delle specie di maggiore interesse. 10 - Neophriseria singula (Staudinger, 1876), §̂̉ (13 mm); 11 Ornativalva heluanensis (Debski, 1913), ठ̂ (11 mm); 12 - Eana nervana (Joannis, 1908), ठ̀ (23 mm); 13 - Stenoptilodes taprobanes (Felder \& Rogenhofer, 1875), ๆ (15 mm); 14 - Merrifeldia garrigae Bigot \& Picard, 1989, đ̊ (16 mm); 15 - Dioryctria simplicella Heinemann, 1863, ô (27 mm); 16 - Cremnophila sedakovella (Eversmann, 1851), † (19 mm); 17 - Ancylolomia inornata Staudinger, 1870, §̂ (23 mm) (foto Théo Léger). 
Caryocolum vicinella (Douglas, 1851) [det. Karsholt] Gambarie, 6.VII.2013 (lux).

Distribuzione geografica: Presente in larga parte dell'Europa fino agli Urali meridionali. In Italia è segnalata per la parte continentale e la Sicilia (Karsholt \& Nieukerken, 2017).

Biologia: Larva su molte Caryophyllaceae, come per esempio Cerastium arvense, Dianthus spergularia, Lychnis alpina, Petrorhagia saxifraga, Silene nutans, ecc.

Stomopteryx basalis (Staudinger, 1876) [det. Karsholt]

Monte Ulis, 11.VII.2013 (lux).

DistribuZIONE GEOGRAFICA: Specie mediterranea conosciuta di Portogallo, Francia, Corsica, Italia, Sicilia, Malta, Creta, Cipro (Karsholt \& Nieukerken, 2017). Prima segnalazione per la Calabria.

Biologia: Sconosciuta.

Stomopteryx remissella (Zeller, 1847) [det. Karsholt] Monte Ulis, 10-11.VII.2013 (lux).

DistribuZIONE GEOGRAFICA: Diffusa in modo discontinuo Europa, dalla regione mediterranea fino alla Svezia a Nord e alla Russia a Est. In Italia è presente in tutta la parte continentale e in Sicilia (Baldizzone et al., 2013).

BiologiA: Larva su Acetosa pratensis, e Crinitaria linosyris.

Syncopacma polychromella (Rebel, 1902) [det. Karsholt] Gambarie, 6.VII.2013 (lux).

Distribuzione geografica: Isole Canarie, Penisola Iberica, Italia, Sicilia, Austria, Rep. Ceca, Croazia, Macedonia, Grecia, Creta, Malta; presente in Africa settentrionale, Vicino e Medio Oriente, Asia centrale, Sudan, Namibia, Sudafrica.

Biologia: La larva si sviluppa su diverse leguminose tra cui Cytisus scoparius.

Crossobela trinotella (Herrich-Schäffer, 1856) [det. Karsholt]

Foce della Fiumara Amendolea, 9.VII.2013 (lux).

DisTRIBUZIONE GEOGRAFICA: Diffusa nella regione mediterranea e parzialmente nell'Europa centrale, fino alla Russia meridionale; presente in Asia Centrale. Per l'Italia è segnalata della penisola, di Sicilia e Sardegna (Karsholt \& Nieukerken, 2017).
Biologia: Si sviluppa su Brassicaeae, come Cheiranthus cheiri, Erysimum durum.

Anarsia spartiella (Schrank, 1802) [det. Karsholt] Monte Ulis, 11.VII.2013 (lux).

DistribuZione GeOgrafiCA: Gran Bretagna, Europa centrale e meridionale, Svezia. Conosciuta di tutta Italia, isole comprese (Huemer \& Karsholt, 1995).

Biologia: Larva su Cytisus scoparius, Genista tinctoria, Ulex europaeus e altre Fabaceae.

Nothris verbascella (Denis \& Schiffermüller, 1775) [det. Nel]

Monte Ulis, 7-8.VII.2013 (lux). det. Nel

DistribUZIONE GEOGRAFICA: Largamente diffusa nella maggior parte d'Europa. Per l'Italia si hanno dati di tutta la parte continentale, Sicilia e Sardegna comprese (Baldizzone et al., 2013).

Biologia: Vive a spese di varie specie del genere Verbascum, attorno alle cui foglie la larva intesse una tela.

Nothris congressariella (Bruand, 1858) [det. Nel] Monte Ulis, 8.VII.2013 (lux).

DistribuZione geografica: Gran Bretagna, Penisola Iberica, Francia, Svizzera, Italia, Balcani, Grecia. Conosciuta di tutta Italia, isole comprese (Baldizzone et al., 2013).

Biologia: Larva su Scrophularia scorodonia.

Brachmia blandella (Fabricius, 1798) [det. Karsholt] Cucullaro, 12.VII.2013 (lux); Foce della Fiumara Amendolea, 9.VII.2013 (lux).

DistribuZione GeOGRAFICA: Largamente diffusa in Europa, in Italia è nota di tutta la parte continentale e della Sicilia (Baldizzone et al., 2013).

Biologia: Conosciuta in modo incompleto; è stato scoperto che la larva si nutre delle foglie di Ulex europaeum.

Tuta absoluta (Meyrick, 1917) [det. Karsholt] Foce della Fiumara Amendolea, 9.VII.2013 (lux). DistribuZIONE GEOGRAFICA: Originaria dell'America meridionale, la specie sta diffondendosi rapidamente in Europa, dove è stata segnalata dapprima in Spagna, poi in Francia, Italia, Malta, Grecia e in Turchia e anche in Africa settentrionale. Segnalata di varie regioni dell'Africa tropicale e dell'India.

BIOLOGIA: Si sviluppa sul pomodoro, cui arreca danni 
gravi e su altre piante spontanee della famiglia Solanaceae.

\section{Tortricidae}

Aethes francillana (Fabricius, 1794) [det. Nel]

Monte Ulis, 7.VII.2013 (lux).

DistribuZIONE GEOGRAFICA: Largamente diffusa in quasi tutta Europa, Isole Canarie, Africa settentrionale, Asia Minore, Medio Oriente, Asia fino all'Afghanistan e Siberia occidentale. Per l'Italia è conosciuta di molte regioni della parte continentale e della Sardegna (Trematerra, 2003).

Biologia: La larva si sviluppa su varie ombrellifere.

Cochylidia rupicola (Curtis, 1834)

Gambarie, 6.VII.2013 (lux).

DistribuZione geograficA: Asia Minore, Transcaucaso ed Europa, dove è largamente diffusa. In Italia è nota di Valle d'Aosta, Piemonte, Lombardia, Veneto, Trentino-Alto Adige, Friuli-Venezia Giulia, EmiliaRomagna, Toscana, Umbria, Abruzzo, Sardegna (Trematerra, 2003). Nuova per la Calabria.

Biologia: Larva segnalata su Agrimonia spp., Chrysocoma linosyris, Eupatorium cannabinum, Lycopus europaeus.

Acleris hastiana (Linnaeus, 1758)

Foce della Fiumara Amendolea, 9.VII.2013 (lux)

DistribuZIONE GEOGRAFICA: Diffusa in quasi tutta Europa, Marocco, Asia Minore, si estende ad Est fino all'Estremo Oriente Russo; presente anche in America settentrionale e nella Regione Neotropicale. In Italia è conosciuta di Piemonte, Lombardia, Trentino-Alto Adige, Liguria, Toscana, Molise, Basilicata e Sardegna (Trematerra, 2003). Nuova per la Calabria.

Biologia: Polifaga su molte piante arboree, e arbustive, tra cui varie specie di Populus, Prunus e Salix.

Eana incanana (Stephens, 1852)

Gambarie, 6.VII.2013 (lux).

DistribuZione Geografica: Presente in quasi tutta Europa, Transcaucasia, Siberia, Estremo Oriente Russo. In Italia è segnalata di Valle d'Aosta, Lombardia, Trentino-Alto Adige, Toscana, Basilicata e Sicilia (Trematerra, 2003). Nuova per la Calabria.

Biologia: Come piante alimentari sono conosciute Chrysanthemun spp., Hyacinthoides spp., Leucanthemum, Salix spp., Scilla spp., Vaccinium spp., ecc.
Eana nervana (Joannis, 1908) [det. Nel] (Fig. 12) Vallone delle Serre, 7.VII.2013 (lux).

Distribuzione geografica: Conosciuta solo di Spagna, Francia e Svizzera. Specie nuova per l'Italia.

Biologia: Sconosciuta.

Archips podana (Scopoli, 1763) [det. Nel]

Gambarie, 6.VII.2013 (lux).

DistribuZIONE GEOGRAFICA: Tutta Europa, introdotto nell'America settentrionale. In Italia è molto diffusa in tutto il territorio (Trematerra, 2003).

BiologiA: Specie polifaga.

Archips xylosteana (Linnaeus, 1758) [det. Nel]

Gambarie, 6.VII.2013 (lux).

DistribuZione GEOGRAFICA: Tutta Europa, dall'Asia Minore fino al Giappone. In Italia è molto diffusa, con dati per quasi tutte le regioni (Trematerra, 2003). Nuova per la Calabria.

Biologia: Largamente polifaga, su piante dei generi Abies, Acer, Alnus, Betula, Castanea, Citrus, Cornus, Corylus, Crataegus, Fagus, Fraxinus, Lonicera, Malus, Myrica, Populus, Prunus, Pyrus, Quercus, Rhamnus, Salix, Sorbus, Tilia, Ulmus.

Ptycholoma lecheana (Linnaeus, 1758)

Vallone delle Serre, 7.VII.2013 (lux).

DistribuZIONE GEOGRAFICA: Specie Paleartica nota di tutta Europa, Asia Minore, Caucaso, Urali Asia centrale, Siberia, Transbaikalia, Cina, Estremo Oriente russo, Corea e Giappone; in Italia è conosciuta di Piemonte, Lombardia, Trentino-Alto Adige, Friuli-Venezia Giulia, Liguria, Emilia-Romagna, Toscana, Umbria, Lazio, Abruzzo, Molise e Sicilia (Trematerra, 2003). Nuova per l'Italia meridionale.

Biologia: Larva su specie di vari generi, di piante arboree, tra cui Abies, Acer, Larix, Populus, Quercus, Sorbus, Tilia, Ulmus, ecc.

Pandemis cerasana (Hübner, 1786) [det. Nel] Gambarie, 6.VII.2013 (lux).

DistribuZione Geografica: Subregione Paleartica, ampiamente diffusa in tutta l'Europa. In Italia è nota per Valle d'Aosta, Piemonte, Lombardia, Veneto, Trentino-Alto Adige, Friuli-Venezia Giulia, Liguria, Emilia-Romagna, Lazio, Abruzzo, Molise, Calabria (Trematerra, 2003).

Biologia: Larva dal comportamento largamente polifago. 
Clepsis consimilana (Hübner, 1817)

Monte Ulis, 10.VII.2013 (lux); Cucullaro, 12.VII.2013 (lux).

DisTRIBUZIONE GEOGRAFICA: Quasi tutta l'Europa, Marocco, Asia Minore, Libano; presente in Madagascar e America settentrionale a causa d'introduzione accidentale. Diffusa in tutta Italia, Sicilia e Sardegna comprese (Trematerra, 2003).

Biologia: Larva polifaga su varie piante, quali Carpinus betulus, Crataegus spp., Ligustrum vulgare, Malus sylvestris, Polygonum spp., Syringa spp., Ulmus spp.

Piniphila bifasciana (Haworth, 1811) [det. Nel] Gambarie, 6.VII.2013 (lux).

DistribuZIone GEOGRAFICA: Diffusa in gran parte dell'Europa, si estende ad Est fino all'Estremo Oriente Russo, Corea e Giappone. In Italia è segnalata per Valle d'Aosta, Lombardia, Trentino-Alto Adige, Liguria, Lazio, Puglia, Sicilia e Sardegna (Trematerra, 2003). Nuova per la Calabria.

Biologia: Larva su Abies spp., Pinus spp., Rhododendron spp., Vaccinium spp.

Epinotia thapsiana (Zeller, 1847) [det. Nel]

Monte Ulis, 7, 11.VII.2013 (lux).

DisTRIBUZIONE GEOGRAFICA: Quasi tutta Europa (manca in Gran Bretagna, Scandinavia e parte dell'Europa centrale e Balcani), Canarie, Marocco, Asia Minore, Transcaucasia, Iran, Kazakistan, Turkmenistan, Tagikistan, Asia centrale, Corea e Siberia occidentale e meridionale. In Italia si hanno dati per Valle d'Aosta, Piemonte, Veneto, Trentino-Alto Adige, Friuli-Venezia Giulia, Toscana, Lazio, Abruzzo, Molise, Basilicata, Calabria, Sicilia e Sardegna (Baldizzone et al., 2103).

Biologia: La larva vive a spese di Crithmum maritimum, Ferula communis, Foeniculum vulgare, Laserpitium gallicum, Ligusticum lucidum, Thapsia villosa.

Gypsonoma minutana (Hübner, 1799) [det. Nel] Foce della Fiumara Amendolea, 9.VII.2013 (lux) DistribuZione GeOgraficA: Tutta l'Europa, Africa settentrionale, Asia Minore, Asia fino alla Cina, Giappone, America settentrionale. In Italia è conosciuta di Piemonte, Lombardia, Trentino-Alto Adige, Liguria, Emilia Romagna, Toscana, Molise, Basilicata, Calabria e Sicilia (Trematerra, 2003).

BIOLOGIA: La larva si sviluppa su varie specie dei generi Populus e Salix.
Gypsonoma aceriana (Duponchel, 1843) [det. Nel] Foce della Fiumara Amendolea, 9.VII.2013 (lux) Distribuzione geograficA: Tutta l'Europa, Africa settentrionale, Asia Minore, Asia occidentale, Iraq. In Italia è conosciuta di Valle d'Aosta, Piemonte, FriuliVenezia Giulia, Emilia-Romagna, Toscana, Umbria, Lazio, Abruzzo, Molise, Campania, Basilicata, Sicilia e Sardegna (Trematerra, 2003). Nuova per la Calabria. Biologia: Larva su Acer spp., Betula spp., Erica spp., Populus spp., Vaccinium spp.

Cydia fagiglandana (Zeller, 1841) [det. Nel] Gambarie, 6.VII.2013 (lux); Vallone delle Serre, 7.VII.2013 (lux).

DistribuZIONE GEOGRAFICA: Diffusa in tutta l'Europa e presente a Madeira, Africa settentrionale, Asia Minore, Siria, Caucaso, Transcaucasia e Turkestan. In Italia è nota per molte regioni, comprese le isole ed è probabilmente diffusa in tutto il territorio nazionale (Baldizzone et al., 2013).

Biologia: Larva su Castanea sativa, Corylus avellana, Fagus sylvatica e Quercus spp. Probabilmente bivoltina, con adulti da maggio a settembre.

Grapholita molesta (Busck, 1916) [det. Nel]

Vallone delle Serre, 7.VII.2013 (lux).

DistribuZIone GeOgraficA: Quasi tutta Europa, Marocco, Medio Oriente, Asia fino al Giappone, America settentrionale, Australia, America meridionale. In Italia è presente in tutte le regioni (Trematerra, 2003). BIologia: Polifaga su molte specie della famiglia Rosaceae.

Pammene fasciana (Linnaeus, 1761) [det. Nel] Monte Ulis, 10.VII.2013 (lux).

Distribuzione GEOgraficA: Tutta Europa, Asia Minore, Caucaso, Transcaucasia, Iran. Diffusa in tutta Italia, isole comprese.

Biologia: Larva su Acer spp., Castanea sativa, Fagus sylvatica, Quercus spp.

Strophedra weirana (Douglas, 1850) [det. Nel] Gambarie, 6.VII.2013 (lux).

DistribuZIONE GEOGRAFICA: Buona parte del'Europa (manca nella Penisola Iberica) e Caucaso. In Italia è segnalata di Piemonte, Liguria e Molise (Trematerra, 2003). Nuova per L'Italia meridionale.

Biologia: Si sviluppa su Carpinus betulus, Castanea sativa, Fagus spp. 


\section{Choreutidae}

Anthophila fabriciana (Linnaeus, 1767)

Monte Ulis, 7.VII.2013 (lux).

DisTRIBUZIONE GEOGRAFICA: Largamente diffusa nella Regione Paleartica, segnalata anche per il Canada. Presente in tutta Italia, isole comprese (Baldizzone et al., 2013).

Biologia: Larva su Urtica dioica, Parietaria officinalis, Symphytum tuberosum.

Tebenna micalis (Mann, 1857)

Foce della Fiumara Amendolea, 9.VII.2013 (lux). DistRIBUZIONE GEOGRAFICA: Diffusa in buona parte dell'Europa centrale e orientale, nella regione mediterranea, Caucaso, Isola Canarie, Africa settentrionale, Asia fino alla Cina, Africa tropicale, Australia, America meridionale. In Italia è segnalata del territorio continentale e della Sardegna (Zangheri, 1995).

Biologia: Larva su varie specie di Compositae, quali Inula spp., Pulicaria dysenterica, ecc.

\section{Alucitidae}

Alucita desmodactyla Zeller, 1847 [det. Nel]

Monte Ulis, 10-11.VII.2013 (lux).

DistribuZIONE GEOGRAFICA: Europa centrale, Francia, Spagna, Italia, Grecia, Creta, Russia, Armenia, Tunisia. In Italia è conosciuta di Piemonte, Lazio, Abruzzo, Puglia e Basilicata (Baldizzone et al., 2013). Nuova per la Calabria.

Biologia: Larva su Stachys recta, S. alpina e S. sylvatica.

\section{Pterophoridae [tutti det. Nel]}

Agdistis heydeni (Zeller, 1852)

Monte Ulis, 8.VII.2013 (lux).

Distribuzione Geografica: Portogallo, Spagna, Francia, Corsica, Italia, Polonia, Albania, Bulgaria, Grecia, Creta. In Italia si hanno dati per Piemonte, Toscana, Umbria, Lazio, Abruzzo, Puglia, Basilicata, Calabria, Sicilia e Sardegna (Baldizzone et al., 2013).

Biologia: Larva detritivora nella lettiera, soprattutto di Labiate (dei generi Ballota, Calamintha, Lamium, Origanum, Phlomis, Stachys, ecc.) e altre piante erbacee, come per esempio Artemisia spp.

Agdistis tamaricis (Zeller, 1847)

Foce della Fiumara Amendolea, 9.VII.2013 (lux).
Distribuzione geografica: Penisola Iberica, Francia, Corsica, Italia, Sicilia, Sardegna, Malta, Svizzera, Balcani, Romania, Bulgaria, Grecia, Creta, Israele, Africa settentrionale, Asia Minore, Iran, Afghanistan, Pakistan, Cina, Arabia. In Italia è conosciuta di Veneto, Emilia-Romagna, Marche, Toscana, Lazio, Lucania, Puglia, Sicilia, Sardegna (Prola \& Racheli, 1984). Prima segnalazione per la Calabria.

Biologia: Larva su Tamarix spp.

Amblyptilia acanthadactyla (Hübner, 1813)

Gambarie, 6.VII.2013 (lux); Monte Ulis, 11.VII.2013 (lux).

DistribuZione GeograficA: Largamente diffusa in Europa. In Italia è segnalata di Piemonte, Lombardia, Veneto, Trentino-Alto Adige, Toscana, Emilia Romagna, Lazio, Abruzzo, Puglia, Calabria, Basilicata, Sicilia, Sardegna (Baldizzone et al., 2013).

BiologiA: Larva antofaga e spermatofaga, osservata su erbe dei generi Aquilegia, Calamintha, Chaenorhinum, Lavandula, Mentha, Prasium, Rosmarinus, Stachys, Teucrium, ecc.

Stenoptilodes taprobanes (Felder \& Rogenhofer, 1875) (Fig. 13)

Foce della Fiumara Amendolea, 9.VII.2013 (lux). DistribuZione GeograficA: Penisola Iberica, Francia, Italia, Sicilia, Malta, Bulgaria, Grecia, Creta. In Italia è segnalata solo del Lazio (Prola \& Racheli, 1984). Nuova per l'Italia meridionale.

Biologia: In Europa si sviluppa su Spergularia media.

Crombrugghia laetus (Zeller, 1847)

Foce della Fiumara Amendolea, 9.VII.2013 (lux).

Distribuzione geografica: Portogallo, Spagna, Francia, Corsica, Italia, Bulgaria, ex Jugoslavia, Grecia. In Italia è conosciuta di Valle d'Aosta, Piemonte, Trentino-Alto Adige, Emilia Romagna, Toscana, Lazio, Umbria, Abruzzo, Puglia, Calabria, Sicilia, Sardegna (Baldizzone et al., 2013).

BIOLOGIA: Specie plurivoltina; larva su specie del genere Andryala.

Calyciphora albodactyla (Fabricius, 1794)

Monte Ulis, 8.VII.2013.

DistribuZIONE GEOGRAFICA: Diffusa in buona parte dell'Europa. In Italia è conosciuta di Piemonte, Lombardia, Trentino-Alto Adige, Emilia Romagna, Lazio, 
Abruzzo, Campania, Calabria, Lucania, Puglia, Sicilia (Prola \& Racheli, 1984).

Biologia: Larva su Echinops ritro, Carlina vulgaris, Carlina acanthifolia, Cirsium ferox.

Merrifeldia garrigae Bigot \& Picard, 1989 (Fig. 14) Monte Ulis, 7-8.VII.2013 (lux).

Distribuzione Geografica: Francia meridionale e Corsica. La specie è nuova per l'Italia.

BIOLOGIA: Larva su Rosmarinus officinalis.

\section{Pyralidae}

Hypotia corticalis (Denis \& Schiffermüller, 1775)

Monte Ulis, 8.VII.2013 (lux).

Distribuzione Geografica: Nord Africa, Asia Minore, Siria, Palestina e Canarie. In Italia è citata genericamente per tutto il territorio (Bassi et al., 1995).

BIOLOGIA: La larva sverna a volte per due inverni nei semi di Daucus carota dell'anno precedente (Slamka, 2006).

Bostra obsoletalis (Mann, 1884)

Foce della Fiumara Amendolea, 9.VII.2013 (lux).

DistRIBUZIONE GEOGRAFICA: Specie mediterranea, conosciuta della Penisola Iberica, Francia meridionale, Corsica, Italia, parte dei Balcani, Malta, Isole Canarie. In Italia è segnalata per le regioni del Nord, Sicilia e Sardegna (Bassi et al., 1995). Recentemente è stata segnalata anche in Calabria (Scalercio, 2016a).

BIOLOGIA: Larva su materiale vegetale secco.

Loryma egregialis (Herrich-Schäffer, 1838)

Gambarie, 6.VII.2013 (lux).

Distribuzione Geografica: Presente in Europa in Bulgaria, Corsica, Creta, Francia, Gibilterra, Grecia, Macedonia, Malta, Portogallo, Spagna e Italia (Karsholt \& Nieukerken, 2017), nota anche del Nord Africa, Palestina, Siria e Turchia (Slamka, 2006). In Italia è segnalata per il settentrione e le Isole maggiori (Bassi et al., 1995), e recentemente anche per l'Italia centrale e meridionale (Scalercio, 2016a, 2016b).

BIOLOGIA: Sconosciuta.

Sciota rhenella (Zincken, 1818) [det. Nel] Foce della Fiumara Amendolea, 9.VII.2013 (lux). DistribuZione GeograficA: Nota in quasi tutta l'Europa, in Italia è diffusa con discontinuità in tutto il territorio peninsulare (Scalercio et al., 2016).
Biologia: Le larve si sviluppano su alcune specie di Populus (Leraut, 2014).

Psorosa dahliella (Treitschke, 1832) [det. Nel] Foce della Fiumara Amendolea, 9.VII.2013 (lux). DistRIBUZIONE GEOGRAFICA: Europa meridionale, Marocco e Siria (Leraut, 2014). In Italia è conosciuta per tutto il territorio (Bassi et al., 1995). Prima segnalazione per la Calabria.

BIOLOGIA: Piante alimentari sconosciute.

Phycita roborella (Denis \& Schiffermüller, 1775) [det. Nel]

Monte Ulis, 7.VII.2013 (lux).

DisTRIBUZIONE GEOGRAFICA: Europa centrale fino alla Gran Bretagna, Irlanda e Scandinavia meridionale, Penisola Iberica, Francia, Italia, Sicilia, Malta, Macedonia, Grecia, Russia, Armenia. Segnalata di tutta Italia, isole comprese (Baldizzone et al., 2013)..

Biologia: Larva su piante del genere Quercus, all'occasione anche su Castanea, Malus, Prunus, Pyrus.

Dioryctria simplicella Heinemann, 1863 [det. Nel] (Fig. 15)

Gambarie, 6.VII.2013 (lux).

DistRIBUZIONE GEOGRAFICA: Larga parte dell'Europa centrale e settentrionale, meno comune nella regione mediterranea. Segnalata per l'Italia solo del Piemonte (Baldizzone et al., 2013). Prima segnalazione per l'Italia centro-meridionale.

Biologia: La larva si sviluppa su piante del genere Pinus.

Acrobasis consociella (Hübner, 1813) [det. Nel] Cucullaro, 12.VII.2013 (lux).

Distribuzione GeOgrafica: Conosciuta di tutta Europa. Segnalata di tutta l'Italia, isole maggiori comprese (Bassi et al., 1995).

Biologia: Larva su Quercus spp.

Apomyelois ceratoniae (Zeller, 1839) [det. Nel]

Foce della Fiumara Amendolea, 9.VII.2013 (lux).

DistribuZIONE GEOGRAFICA: Cosmopolita, accidentalmente introdotta in Europa settentrionale (Leraut, 2014). Segnalata di tutta l'Italia, isole maggiori comprese (Bassi et al., 1995).

Biologia: La larva si nutre di frutta secca, risultando dannosa alle coltivazioni (Leraut, 2014). 
Valdovecaria umbratella (Treitschke, 1832) [det. Nel] Monte Ulis, 11.VII.2013 (lux).

Distribuzione Geografica: Presente in Croazia, Francia, Marocco, Turchia e Asia minore, in Italia è nota solo per Calabria e Sicilia (Scalercio, 2016a).

Biologia: Sconosciuta.

Myelois circumvoluta (Fourcroy, 1785)

Monte Ulis, 8.VII.2013 (lux).

DistribuZIONE GEOGRAFICA: Ampiamente diffusa in Europa, in Italia è conosciuta di tutto il territorio, incluse Sicilia e Sardegna (Bassi et al., 1995).

Biologia: Larve su Cirsium e altri cardi (Leraut, 2014).

Cremnophila sedakovella (Eversmann, 1851) [det. $\mathrm{Nel}$ (Fig. 16)

Monte Ulis, 10.VII.2013 (lux).

DistriBuZione GeOgRAFICA: Nota in Europa per Spagna, Svizzera, Austria e Italia dove è conosciuta solo per l'Arco Alpino (Leraut, 2014). Prima segnalazione per l'Italia centro-meridionale.

Biologia: Ignota.

Asalebria florella (Mann, 1862) [det. Nel]

Monte Ulis, 11.VII.2013 (lux).

DistribUZIONE GEOGRAFICA: Soprattutto nei paesi che si affacciano sul Mediterraneo, ma anche in Germania, Balcani, Russia meridionale e Ucraina. In Italia è segnalata per il Piemonte, il meridione e la Sardegna (Baldizzone et al., 2013).

BIologiA: Larva su Genista spp.

Ephestia unicolorella woodiella Richards \& Thomson, 1932 [det. Nel]

Monte Ulis, 10.VII.2013 (lux).

DistribuZIONE GEOGRAFICA: Segnalata in quasi tutta Europa ad eccezione delle regioni più settentrionali, ad Est fino all'Iran, Nord Africa (Leraut, 2014). Sembrerebbe presente in tutta Italia (Bassi et al., 1995), anche se le vicissitudini tassonomiche della sottospecie spingono ad una valutazione prudenziale delle citazioni disponibili.

Biologia: Larva probabilmente su detrito organico (Leraut, 2014).

Ematheudes punctella (Treitschke, 1833) [det. Nel] Cucullaro, 12.VII.2013 (lux).

DistribuZIONE GeOGRAFICA: Presente in larga parte di Europa, ma manca in Gran Bretagna, Scandinavia e
Paesi Baltici. Conosciuta di tutta Italia, isole maggiori comprese (Baldizzone et al., 2013).

Biologia: Non conosciuta.

\section{Crambidae}

Anarpia incertalis (Duponchel, 1832) [det. Nel] Monte Ulis, 11.VII.2013 (lux).

DistribuZIONE GEOGRAFICA: Nota dal Bacino del Mediterraneo fino al Kirghizistan (Leraut, 2014). In Italia è nota per il settentrione e per le Isole Maggiori (Bassi et al., 1995), ma nella collezione del CREA-FL è conservato un esemplare raccolto ad Anchiano, Toscana, il 5.IX.1995, S. Scalercio legit. Prime segnalazioni per l'Italia centro-meridionale.

Biologia: Ignota.

Eudonia lacustrata (Panzer, 1804) [det. Nel]

Monte Ulis, 12.VII.2013 (lux).

DistribuZIONE GEOGRAFICA: Comune in tutta Europa, presente in Africa nord-occidentale, Siria, Anatolia, Iran, Siberia, Cina. In Italia è presente in tutto il territorio (Scalercio, 2016a).

Biologia: La larva vive in varie specie di muschi, come Hypnum cupressiforme, Polytrichum commune, Tortella tortuosa, e altri (Goater et al., 2005).

Eudonia delunella (Stainton, 1849) [det. Nel]

Monte Ulis, 6.VII.2013 (lux).

DistribuZIONE GEOGRAFICA: Presente in buona parte d'Europa, più rara al settentrione, in Nord Africa e nel Caucaso (Leraut, 2014), segnalata per l'Italia centrosettentrionale e la Sardegna (Pinzari et al., 2010). Prima segnalazione per l'Italia meridionale.

Biologia: Larva su licheni e muschi che crescono su frassino, meli e talvolta olmi (Goater et al., 2005).

Eudonia mercurella (Linnaeus, 1758) [det. Nel] Cucullaro, 12.VII.2013 (lux).

DistribuZIONE GeOgRAFiCA: Molto comune in tutta Europa; presente in Africa nord-occidentale, Turchia, Libano, Iran. In Italia è distribuita in tutto il territorio, isole comprese (Baldizzone et al., 2013).

BIOLOGIA: Larva su muschi viventi su tronchi e muri, come Hypnum cupressiforme e Isothecium alopecuroides.

Catoptria spatulelloides Bleszynski, 1965 [det. Bassi] Gambarie, 6.VII.2013 (lux); Vallone delle Serre, 7.VII.2013 (lux). 
DistribuZIONE GEOGRAFICA: Endemismo calabro-lucano. Hartig (1972) ha descritto per Monte Ulis C. parenzani, successivamente messa in sinonimia con questa specie.

BIOLOGIA: Larva sconosciuta.

Catoptria mytilella (Hübner, 1805) [det. Bassi]

Gambarie, 6.VII.2013 (lux).

Distribuzione geografica: Ampiamente diffusa nel Centro e Sud Europa. In Italia è presente praticamente in tutta la penisola (Baldizzone et al., 2013).

BIOLOGIA: Larva nei muschi.

Pediasia contaminella (Hübner, 1796) [det. Bassi] Vallone delle Serre, 7.VII.2013 (lux).

DistribuZione GEOGRAFICA: Specie ampiamente diffusa in Europa, Asia Minore fino alla Siberia meridionale. Tutta l'Italia e isole maggiori (Baldizzone et al., 2013).

Biologia: Larva su Poaceae (generi Festuca e Poa).

Ancylolomia inornata Staudinger, 1870 [det. Bassi] (Fig. 17)

Foce della Fiumara Amendolea, 9.VII.2013 (lux). DistribuZione GeograficA: Nota per Spagna, Portogallo, Italia meridionale, Sicilia, Malta e Nord Africa. È nota una femmina dal Pakistan (Slamka, 2008). In Calabria è già stata segnalata per la costa ionica settentrionale a Rossano Lido (Ippolito, 1987).

BIOLOGIA: Larva ignota (Slamka, 2008).

Eurrhypis pollinalis (Denis \& Schiffermüller, 1775) Serra Juncari, 11.VII.2013.

DistribuZIONE GEOGRAFICA: Diffusa in Europa, in Italia è riportata solo per il territorio continentale centrosettentrionale e per la Sicilia (Baldizzone et al., 2013), ma è citata anche per la Calabria Ulteriore da Costa A. (1863). Questa segnalazione conferma la presenza della specie anche in Italia meridionale dopo oltre 150 anni.

Biologia: Larva su leguminose arbustive come Cytisus, Genista, Ononis (Baldizzone et al., 2013).

Evergestis segetalis (Herrich-Schäffer, 1851)

Gambarie, 6.VII.2013 (lux); Vallone delle Serre, 7.VII.2013 (lux); Monte Ulis, 8.VII.2013 (lux).

DistribuZIone GeograficA: Nota per Francia, Bulgaria, Macedonia, Russia europea, Turchia, Algeria e Iran (Goater et al., 2005). In Italia è conosciuta per il territorio continentale e la Sicilia (Bassi et al., 1995). Nuova per la Calabria.

BIOLOGIA: Larva sconosciuta.

Udea olivalis (Denis \& Schiffermüller, 1775)

Gambarie, 6.VII.2013 (lux).

DistribuZione Geografica: Ad eccezione della Penisola Iberica è diffusa in buona parte dell'Europa e in Asia centrale (Slamka, 2013). In Italia è nota per il centro, il Nord e la Calabria (Scalercio, 2016a).

BIologia: La larva è polifaga su molte piante come Urtica, Senecio, Geum, Stachys, Humulus, Rubus, Sambucus, Lonicera e altre (Slamka, 2013).

Mecyna asinalis (Hübner, 1819)

Gambarie, 6.VII.2013 (lux).

DistribuZIone GeOgRAFICA: Diffusa in Europa meridionale, Irlanda, Gran Bretagna sud-occidentale, molte isole mediterranee, Canarie, Azzorre e Turchia. In Italia è nota con discontinuità in buona parte del territorio, isole comprese (Slamka, 2013).

Biologia: Larva su Rubia peregrina e Crucianella maritima (Slamka, 2013).

Duponchelia fovealis Zeller, 1847 [det. Nel] Foce della Fiumara Amendolea, 9.VII.2013 (lux). DistribUZIONE GEOGRAFICA: Originaria dei tropici e del Bacino del Mediterraneo, è stata importata in Europa settentrionale, dove sopravvive nelle serre, e in Nord America (Slamka, 2013). In Italia è segnalata per quasi tutto il territorio, isole comprese. Già nota in $\mathrm{Ca}$ labria per Curinga (CZ) (NPPO of Italy, 2010).

Biologia: Larva polifaga che attacca anche molte piante ornamentali (Slamka, 2013).

\section{CONCLUSIONI}

Questa pubblicazione faunistica, pur essendo basata sui risultati di una sola settimana di raccolta effettuata con diverse tecniche sull'Aspromonte e alla foce della Fiumara Amendolea, ha permesso di segnalare 159 specie per il territorio esaminato. Di particolare rilievo i rinvenimenti di Eana nervana (Tortricidae) e Merrifeldia garrigae (Pterophoridae) che risultano essere nuove per la fauna italiana. Di rilevante interesse sono anche i rinvenimenti di Opogona omoscopa (Tineidae), Triberta cistifoliella (Gracillariidae), Ornativalva heluanensis e Neophriseria singula (Gelechiidae) che vengono segnalate per la prima volta in Italia continentale. Inoltre, Agonop- 
terix ferocella, A. nervosa (Depressariidae), Elachista dispunctella (Elachistidae), Coleophora alnifoliae, $C$. ptarmicia (Coleophoridae), Monochroa melagonella (Gelechiidae), Dioryctria simplicella, Cremnophila sedakovella (Pyralidae), e Anarpia incertalis (Crambidae) sono nuove per l'Italia centro-meridionale, altre 16 per l'Italia meridionale, e 28 per la Calabria. Il dato relativo alle novità faunistiche a livello regionale è certamente sottostimato in quanto sono state considerate tali solo le specie per le quali abbiamo avuto la possibilità di accertarci che non fossero già state segnalate per la regione, mentre abbiamo generalmente considerato come già segnalate quelle specie per le quali esistono esclusivamente informazioni generiche di presenza in tutto il territorio italiano.

La grande quantità di novità faunistiche rinvenute sono l'evidenza delle scarse conoscenze disponibili sulla fauna di questi insetti per l'Italia in generale e per le regioni meridionali in particolare, dove l'esplo- razione metodica potrebbe in futuro portare ad un notevole incremento della fauna italiana dal momento che alcuni biotopi sono praticamente inesplorati.

\section{RINGRAZIAMENTI}

Ringraziamo la direzione del Parco Nazionale dell'Aspromonte per il permesso ad effettuare raccolte nel territorio del parco, Carlo Cabella (Novi Ligure) e Giuseppe Longo Turri (Verona) per l'aiuto durante le ricerche. Per la determinazione di numerose specie, informazioni e collaborazioni in vario modo, ringraziamo Graziano Bassi (Avigliana), Reinhard Gaedike (Bonn, Germania), Ole Karsholt (Copenaghen, Danimarca), Bernard Landry (Ginevra, Svizzera), Théo Léger (Ginevra, Svizzera), Jacques Nel (La Ciotat, Francia), Erik van Nieukerken (Leiden, Olanda), Paolo Triberti (Negrar), Pier Giuseppe Varalda (Morano sul Po).

\section{BIBLIOGRAFIA}

BALdizzone G., 2014 - Contribuzioni alla conoscenza dei Coleophoridae. CXXIX. Coleophora aspromontis Baldizzone: nuova specie del gruppo di C. lutipennella (Zeller, 1838) (Lepidoptera: Coleophoridae). SHILAP Revista de Lepidopterología, 42: $373-378$

Baldizzone G., 2018 - Lepidoptera Coleophoridae. Fauna d'Italia. LIII. Calderini, Bologna, (in stampa).

Baldizzone G., Cabella C., Fiori F., Varalda P.G., 2013 - I Lepidotteri del Parco Naturale delle Capanne di Marcarolo (Italia, Piemonte, Appennino ligure-piemontese). Memorie dell'Associazione Naturalistica Piemontese, 12, 349 pp.

Baraniak E., Gaedike R., Karsholt O., Triberti P., Zangheri S., 1995 - Insecta Lepidoptera Tineoidea II. In: Minelli A., Ruffo S., La Posta S. (eds.), Checklist delle specie della fauna italiana, 82. Calderini, Bologna.

Bassi G., Passerin D’Entreves P., Speidel W., Zangheri S., 1995 - Insecta Lepidoptera Pyraloidea. In: Minelli A., Ruffo S. \& La Posta S. (eds.), Checklist delle specie della fauna italiana, 87. Calderini, Bologna.

BASsi G., Nel J., 2015 - Due microlepidotteri nuovi per la fauna d'Italia (Lepidoptera: Glyphipterigidae, Acrolepiinae; Tineidae, Hieroxestinae). Bollettino della Società entomologica Italiana, 147 (3): 135-136.

Braggio S., Triberti P., 2011 - Remarks on some families of leaf-mining Microlepidoptera from central-southern Sardinia, with some ecological considerations (Lepidoptera: Nepticulidae, Bucculatricidae, Gracillariidae), pp. 767-781. In: NARDI G., Withmore D., Bardiani M., Birtele D., Mason F., Spada L. \& Cerreti P. (eds), Biodiversity of Marganai and Montimannu (Sardinia). Research in the framework of the ICP Forests network. Conservazione Habitat Invertebrati, 5. Cierre edizioni, Sommacampagna, Verona.

Costa A., 1863 - Nuovi studii sulla entomologia della Calabria Ulteriore. Napoli, Stamperia del Fibreno, 80 pp.

Costa A, 1881. Relazione di un viaggio nelle Calabrie per ricerche zoologiche fatto nella state del 1876. Accademia delle Scienze Fisiche e Matematiche.

Costa O. G., 1832-1836 - Fauna del Regno di Napoli. Lepidotteri. Parte prima. Lepidotteri Diurni, Crepuscolari ed alcune famiglie de' Notturni. Dai torchi del Tremater, Napoli, 204 pp.

Curò A., 1880 - Saggio di un catalogo dei lepidotteri d'Italia. Bullettino della Società entomologica Italiana, 12: 51-92, 153-191.

Curò A., 1883 - Saggio di un catalogo dei lepidotteri d'Italia. Bullettino della Società entomologica Italiana, 15: 1-124.

De Prins J., De Prins W., 2018 - Global taxonomic database of Gracillariidae (Lepidoptera). Worls Wide Web electronic publication (http://www.gracillariidae.net) (ultimo accesso: marzo 2018).

De Prins J., Davis D. R., De Coninck E., Sohn J., Triberti P., 2013 - Systematics, phylogeny and biology of a new genus of Lithocolletinae (Lepidoptera: Gracillariidae) associated with Cistaceae. Zootaxa, 3741 (2): 201- 227.

Elsner G., Huemer P., Tokár Z., 1999 - Die Palpenmotten (Lepidoptera, Gelechiidae) Mitteleuropas. Bratislava, 208 pag. 
Emmet A.M., Watkinson I.A. \& Wilson M.R., 1985 - Gracillariidae. In: Heath J. \& Emmet A.M. (eds), The Moths and Butterflies of Great Britain and Ireland, 2. Harley Books, Colchester, Essex: 244-363.

Gaedike R., Hattenschweiler P., Triberti P., Zangheri S., 1995 - Lepidoptera Tineoidea I. In: Minelli A., Ruffo S. \& La Posta S. (eds.), Checklist delle specie della fauna italiana, 81. Calderini, Bologna.

Gaedike R., Mally R., 2011 - On the taxonomic status of Cephimallota angusticostella (Zeller) and C. crassiflavella Bruand (Tineidae). Nota lepidopterologica, 34 (2): 115-130.

Goater B., Nuss M., Speidel W., 2005 - Microlepidoptera of Europe. Pyraloidea I: 304 pp. Apollo Books, Stenstrup.

GozmÁny L. 2008 - Symmocidae. In: R. Gaedike (ed.), Microlepidoptera Palaearctica 13: 558 pp. Goecke \& Evers, Keltern.

Hartig F., 1972 - Contibuto alla conoscenza e distribuzione della Crambine (Lep. Pyralidae) in Italia. Entomologica, 8: 125131.

Huemer P., Karsholt O., 1995 - In: Baldizzone G., Gozmány L., Huemer P., Karsholt O., Lvovsky A., Parenti U., Passerin D’Entrèves P., Riedl, T., Varalda P.G., Zangheri S., 1995. Insecta Lepidoptera Gelechioidea. In: Minelli A., RufFo S. \& LA Posta S. (eds), Checklist delle specie della fauna italiana, 83. Calderini, Bologna.

Huemer P., Karsholt O., 2010 - Gelechiidae II (Gelechiinae: Gnorimoschemini). In P. Huemer, O. Karsholt \& M. Nuss (eds.): Microlepidoptera of Europe 6: 1-586. Apollo Books, Stenstrup.

IpPolito R., 1987 - Crambini dell'Italia meridionale (Lepidoptera - Pyralidae - Crambinae). Entomologica, 22: 137-155.

JAECKH E., 1972 - Die Gattung Pseudatemelia Rebel, 1910 (Lepidoptera, Oecophoriidae). Entomologica, 8: 133-140.

Karsholt O., NieuKerken E.J., 2017 - Lepidoptera, Moths. Fauna Europaea version 2.6.2, https://fauna-eu.org/ (ultimo accesso 09 maggio 2018).

Koster J.S., Sinev S.Yu., 2003 - Momphidae, Batrachedridae, Stathmopodidae, Agonoxenidae, Cosmopterigidae, Chrysopeleidae. In: Huemer P., Karsholt O., LuneBorg L. (eds.). Microlepidoptera of Europe 5, 387pp.

KAILA L., 2015. The Elachista dispunctella (Duponchel) complex (Lepidoptera, Elachistidae) revisited, with exceptional level of synonymy. Zootaxa, 3980 (3): 301-358.

Karsholt O., Razowski J. (Eds.). (1996) - The Lepidoptera of Europe: a distributional checklist. Brill Academic Pub.

Karsholt O., Rutten, T., 2005 - The genus Bryotropha Heinemann in the Western Palaearctic (Lepidoptera: Gelechiidae). Tijdschrift voor Entomologie, 148: 77-207.

Leonetti F.L., Greco S., Infusino M., ScAlercio S., 2018 - Contributo alla conoscenza dei Gelechioidea dell'Italia meridionale con particolare riferimento ad ambienti forestali (Lepidoptera Chimabachidae, Elachistidae, Oecophoridae, Peleopodidae, Stathmopodidae). Bollettino della Società entomologica Italiana [in stampa].

LeRAut P.J.A., 2014 - Moths of Europe, Pyralids 2: 441 pp. N.A.P. Editions, Verrières-le-Buisson.

MARIANI M., 1943 - Fauna Lepidopterorum Italiae. Parte I. Catalogo ragionato dei Lepidotteri d'Italia. Giornale di Scienze Naturali ed Economiche, Palermo, 42 (1940-1941): 1-237.

Mineldi A., Ruffo S., LA Posta S., 1995 - Checklist delle specie della fauna italiana. Calderini, Bologna.

NPPO of Italy, 2010 - Duponchelia fovealis detected in Calabria. EPPO Reporting Service, 2010 (10): 2010/185.

PAREnti U., 2000 - A guide to the Microlepidoptera of Europe. Guide I. 2000. Museo Regionale di Scienze Naturali Torino, $426 \mathrm{pp}$.

PinZari M., PinZari M., Zilli A., 2010 - Deep lepidopterological exploration of Mt Cagno and surroundings (Central Italy), a restricted mountain massif and hotspot for butterfly and moth diversity (Lepidoptera). Bollettino dell'Associazione Romana di Entomologia, 65 (14): 3-383.

Prola C., Racheli T., 1984 - An annotated list of Italian Pterophoridae (Lepidoptera). Atalanta, 15: 305-337.

Riedl T., 1995 - In: Baldizzone G., Gozmány L., Huemer P., Karsholt O., Lvovsky A., Parenti U., Passerin d’Entrèves P., Riedl,T., Varalda P.G., Zangheri S., 1995. Insecta Lepidoptera Gelechioidea. In: Minelli A., Ruffo S. \& La Posta S. (eds), Checklist delle specie della fauna italiana, 83. Calderini, Bologna.

Scalercio S., 2009 - Messa a punto delle conoscenze sugli Psychidae di Calabria, Italia meridionale (Lepidoptera Tineoidea). Bollettino della Società entomologica Italiana, 141(3): 163-178.

SCALERCIO S., 2016a - Interessanti novità faunistiche sui Pyraloidea dell'Italia meridionale con particolare riferimento agli ambienti forestali (Lepidoptera: Pyralidae, Crambidae). SHILAP Revista de Lepidopterología, 44(175): 433-442.

SCALERCio S., 2016b - Contributo alla conoscenza dei microlepidotteri del Parco Nazionale dell'Arcipelago Toscano (Lepidoptera). Rivista del Museo Civico di Scienze Naturali "E. Caffi”, Bergamo, 29: 105-122.

Scalercio S., LuZzi G., LAUdati M., 2015 - Nuovi reperti per la fauna microlepidotterologica degli ambienti forestali del Parco Nazionale della Sila, area MAB Unesco (Lepidoptera Yponomeutoidea, Gelechioidea). Bollettino della Società entomologica Italiana, 147 (2): 79-84.

Scalercio S., Urso S., Greco S., Infusino M., 2016 - Alcune specie di Pyraloidea nuove per la fauna dell'Italia meridionale (Lepidoptera, Pyralidae, Crambidae). Bollettino della Società entomologica Italiana, 148(2): 71-74. 
SCHMID J., 2011 - Teleiopsis laetitiae sp. n. and Teleiopsis lindae sp. n., two hitherto overlooked mountainous European species (Gelechiidae: Teleiodini). Nota lepidopterologica, 33 (2): 271-283.

Slamka F., 2006 - Pyraloidea of Europe (Lepidoptera) 1. Pyralinae, Galleriinae, Epipaschiinae, Cathariinae \& Odontiinae: 128 pp. Slamka, Bratislava.

Slamka F., 2008 - Pyraloidea of Europe (Lepidoptera) 2. Crambinae \& Schoenobiinae: 224 pp. Slamka, Bratislava.

Slamka F., 2013 - Pyraloidea of Europe (Lepidoptera) 3. Pyraustinae \& Spilomelinae: 357 pp. Slamka, Bratislava.

TokÁr Z., Lvovsky A., Huemer P., 2005 - Die Oecophoridae s. 1. (Lepidoptera) Mitteleuropas. Bratislava.

ToKÁR Z., Buschmann F., 2012 - Wegneria panchalcella (Staudinger, 1871), new records of Tineidae for Hungary and Central Europe (Lepidoptera: Tineidae). Microlepidoptera.hu 5: 39-42.

Trematerra P., 2003 - Catalogo dei Lepidoptera Tortricidae della fauna italiana: geonemia, distribuzione in Italia, note biologiche, identificazione. Bollettino di Zoologia agraria e Bachicoltura, Ser. II, 35 (suppl. 1): 1-270.

TREmaterra P., 2004 - Note su undici lepidotteri Tortricidi nuovi per la fauna italiana.- Bollettino di Zoologia Agraria e Bachicoltura, (2) 36 (2): 221-228.

Triberti P., Longo Turri G., AdAmi R., Zanetti A., 2017 - I Lepidotteri della "Palude del Busatello". Memorie della Società Entomologica Italiana 94: 91-135

Whitebread, S. 1995 - In: Karsholt O., Nieukerken E. J. van, Whitebread S., Zangheri S. 1995. Insecta Lepidoptera Micropterigidae. In: Minelli A., Ruffo S. \& La Posta S. (eds), Checklist delle specie della fauna italiana, 80. Calderini, Bologna.

ZANGHERI S., 1955a - I Lepidotteri del promontorio del Gargano. Commentationes Pontificiae Academiae Scientiarum, 17: 1731.

ZANGHERI S., 1955b - Le attuali conoscenze sui Lepidotteri del Promontorio del Gargano e delle Isole Tremiti, con osservazioni sulle specie a distribuzione transadriatica. Memorie di Biogeografia Adriatica, 3: 245-298.

ZANGHERI S., 1960 - Ricerche faunistiche e zoogeografiche sui Lepidotteri delle Puglie e della Basilicata. Memorie della Società Entomologica Italiana, 39: 5-35.

Zangheri S., 1995 - In: Balletto E., Camporesi S., Cassulo L.A., Fiumi G., Karsholt O., Zangheri S., 1995. Insecta Lepidoptera Cossoidea, Sesioidea, Zygaenoidea, Choreutoidea. In: Minelli A., Ruffo S. La Posta S. (eds), Checklist delle specie della fauna italiana, 84. Calderini, Bologna.

Zeller-LuKashort H.C, Kurz M.E., Lees D.C., KurZ M.A., 2007 - A review of Micropterix Hübner, 1825 from northern and central Europe (Micropterigidae). Nota lepidopterologica, 30 (2): 235-298. 